\title{
Vector Wave Digital Filters and their Application to Circuits with Two-Port Elements
}

\author{
Alberto Bernardini, Member, IEEE, Paolo Maffezzoni, Senior Member, IEEE, \\ Augusto Sarti, Senior Member, IEEE
}

\begin{abstract}
Wave Digital Filters (WDFs) turn circuits into networks of input-output relationships that can be computed in an explicit fashion. This is done through a linear port-wise mapping of Kirchhoff variables into pairs of incident-reflected waves introducing one scalar free parameter per port, called reference port resistance. Parameters are then used to eliminate the implicit equations relating wave variables, referred to as delay-free-loops. Unfortunately, this methodology can only be applied under strong linearity and topological conditions. This manuscript presents an extension of the WDF formalism involving a novel "crossport" vector definition of waves, whose reference resistance is a matrix of free parameters. This generalization greatly simplifies the WDF implementation of circuits with two-port elements, such as operational amplifiers. It allows us to derive wave-based descriptions of elements such as nullors, for which no scattering relation is available in the literature. Moreover, it enables a full adaptation of a wide class of two-port elements, thus avoiding the delay-free-loops that would otherwise form in traditional WDFs. This new formalism allows us to implement a wider range of circuits with two-port elements in a modular fashion, since the topology and the elements can be modeled independently.
\end{abstract}

Index Terms-Wave Digital Filters, Scattering Matrices, Junctions, Adaptors, Connection Networks.

\section{INTRODUCTION}

W AVE Digital Filter (WDF) theory [1] was originally developed in the '70s by A. Fettweis with the purpose of designing pseudopassive digital filters starting from reference passive analog circuits [2]. WDFs rely on a port-wise linear mapping of Kirchhoff pairs of variables (voltage and current) into pairs of wave variables (incident and reflected waves) with the introduction of a scalar free parameter per port called reference port resistance. Combined with an appropriate stable discretization strategy, such as the trapezoidal method, this turns each circuit element into a Wave Digital (WD) block described by an explicit input-output relationship [3]. At the same time, the interconnection topology is turned into an interconnection of scattering junctions [4]. The reference circuit is therefore modeled as an interconnection of WD blocks, which turns out to be characterized by implicit relations between wave variables, called delay-free-loops. According to WDF principles [1], we can actively eliminate such implicit relations by properly setting the free parameters introduced in the WD domain through a process called "adaptation" that eliminates local instantaneous dependencies between incident and reflected wave variables. Unfortunately, only for a class of

A. Bernardini, P. Maffezzoni and A. Sarti are with the Dipartimento di Elettronica, Informazione e Bioingegneria (DEIB); Politecnico di Milano, Piazza L. Da Vinci 32, 20133 Milano, Italy (e-mail: [alberto.bernardini,paolo.maffezzoni,augusto.sarti]@polimi.it). circuits (linear circuits or certain circuits containing a single nonlinearity) it is possible to eliminate all delay-free-loops, and implement the circuit in a fully "explicit" fashion (i.e., without the need of iterative solvers) $[5]-[\overline{8}]$ while using stable discretization methods, such as the trapezoidal method or the backward Euler method [3]. In fact, when dealing with multiple nonlinearities WD implementations typically involve iterative solvers [3], [9]-[13]. Even in this case, however, do WD methods offer some advantages over simulation techniques that are entirely developed in the "Kirchhoff domain". The main reason is that WDF principles allow us to model the topology and the elements of a circuit in a rather modular fashion, employing input-output blocks connected through port connections. Exploiting this fact, recent extensions of WDF principles proved particularly promising and efficient for applications of circuit simulation, when compared with traditional circuit simulation software, as they do not necessarily require the construction of large Jacobian matrices and multivariate Newton-Raphson solvers [3], [10], [12]-[14] whose size is in the order of the number of nodes of the reference circuit.

In the WD domain, a one-port circuit element is modeled as a scattering relation derived from its constitutive equation in the Kirchhoff domain. The reflected wave is, in fact, expressed as a function of the incident wave and the introduced free parameter [1]. Topological connections, instead, are modeled as $N$-port junctions, usually referred to as adaptors, which are characterized by $N \times N$ scattering matrices [4], [15]-[18]. A topological graph description of a circuit can be obtained using the so-called $\mathcal{S P Q R}$-tree decomposition [19]; the resulting tree structure maps circuit elements onto $\mathcal{Q}$ nodes; series connections onto $\mathcal{S}$ nodes; parallel connections onto $\mathcal{P}$ nodes; and connections that are neither series nor parallel (triconnected components) onto $\mathcal{R}$ nodes. WDFs are traditionally designed to match the morphology of the corresponding $\mathcal{S P Q R}$-tree structure: $\mathcal{S}$ and $\mathcal{P}$ nodes are implemented with series and parallel adaptors, respectively; while $\mathcal{R}$ nodes are realized using $\mathcal{R}$-type adaptors [19]. Several recent publications are focused on the modeling of $\mathcal{R}$-type adaptors [17], [18], [20][23]. In [18] the meaning of $\mathcal{R}$-type adaptor was generalized to the concept of connection network, which denotes a junction whose role is to connect elements or other networks together. A connection network could represent a simple "wiring" of elements, but it could also embed linear reciprocal or nonreciprocal multi-ports such as multi-winding transformers, nullors, or controlled sources [17]. A general method for implementing reciprocal as well as non-reciprocal connection 
networks in the WD domain is presented in [17], while a less computationally expensive approach for the WD realization of reciprocal connection networks is presented in [18]. We recall that arbitrary purely topological connection networks (i.e., wire connections) are inherently reciprocal and can all be modeled as shown in [18]. The main reasons why linear multi-ports are sometimes embedded into $\mathcal{R}$-type adaptors are related to computability. In fact, connecting multi-port linear elements to adaptors might introduce delay-free-loops. For example, when both ports of a two-port linear element are connected to the same multi-port junction a double delay-free-loop passing through the element and the junction always arises, and it cannot be eliminated even in the case in which both ports of the two-port element are locally made reflection-free [17], [24]. Moreover, scattering relations of many relevant linear multi-ports, such as nullors or controlled sources, cannot be modeled using the traditional WDF formalism. Incorporating linear multi-ports within the junction is an effective way to eliminate such computability problems, but it actually undermines the separation, typical of traditional WDF modeling, between topological adaptors and circuit elements [1].

In this manuscript we present an extension of the traditional port-wise WDF formalism that employs novel vector definitions of waves, involving cross-port reference resistances in the form of $2 \times 2$ matrices of free parameters. We show that this generalization of the WDF theory brings considerable advantages in the implementation of circuits with twoports, such as gyrators, controlled sources or operational amplifiers (opamps). In fact, it allows us to derive wavebased descriptions of elements, such as nullors or controlled sources, for which a scattering relation could not be defined otherwise. It also enables to connect a wide class of linear two-ports to $\mathcal{R}$-type adaptors without ending up with delayfree-loops. The proposed approach, therefore, solves many of the computability problems that arise when modeling circuits with complicated topologies and multi-port elements, without having to embed the multi-ports within $\mathcal{R}$-type adaptors and maintaining a clear separation between the topological information and the constitutive equations of the elements, just like in WD structures with one-ports.

The manuscript is organized as follows. Section II provides a background on the classical scalar definition of waves in WDFs and on the corresponding WD modeling of a generic two-port element. It then introduces a novel vector definition of wave variables involving a reference two-port resistance. Section III applies such a definition to the WD modeling of the same generic two-port considered in Section II] highlighting its advantages. General conditions for performing the full adaptation of a two-port WD element, i.e., making the pair of ports reflection-free, are provided. Energetic properties of a WD two-port element based on the proposed vector definition of waves are also analyzed. The six sections from IV to IX present detailed WD models of all the six families of linear resistive two-ports [25] as special cases of the general model discussed in Section III. WD models of specific resistive twoports are also discussed as examples, like voltage-controlled or current-controlled sources, the gyrator, and the nullor. Section $\mathrm{X}$ shows how WD topological junctions based on mixed scalar and vector definitions of wave variables can be designed. Closed-form formulas for the formation of the scattering matrices are provided, along with some considerations on their properties. We also show that a pair of ports of a WD junction characterized by a vector definition of waves can be made reflection-free, which is something that could not be done in the case of traditional WDFs. This means that, by properly selecting the free parameters, it is possible to set a $2 \times 2$ block of diagonal entries of the scattering matrix to zero. Section XI proves the effectiveness of the proposed approach by discussing different WD realizations of two circuits: an active band-pass filter with one opamp and a phono preamplifier with two opamps. Different two-port models of op-amps are considered: the nullor-based model, a model with an ideal controlled source, and a model with a resistive controlled source. A comparison between the WD structures obtained with the proposed approach and those obtained with the method in [17] is provided; the advantages brought by the proposed technique are highlighted both in terms of computational cost and in terms of modularity. Section XII concludes this manuscript.

\section{SCALAR AND VECTOR WAVES}

\section{A. Background: Scalar Definition of Wave Variables with Reference One-Port Resistances}

Let us consider a generic two-port circuit element. In the Kirchhoff domain, port $j$ of the element, with $j \in\{1,2\}$, is characterized by a port voltage $v_{j}$ and a port current $i_{j}$, as shown in Fig. 1 .

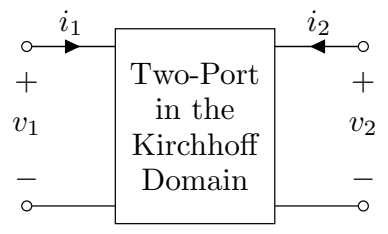

Fig. 1. Generic two-port in the Kirchhoff domain.

In traditional WDFs based on voltage waves, the pair of WD variables at port $j$ is expressed with the following scalar definition [1]

$$
a_{j}=v_{j}+Z_{j j} i_{j} \quad, \quad b_{j}=v_{j}-Z_{j j} i_{j},
$$

where $a_{j}$ is the wave incident to the element, $b_{j}$ is the wave reflected by the element and $Z_{j j} \neq 0$ is a real scalar free parameter, usually called reference port resistance and here renamed as reference one-port resistance.

$$
v_{j}=\frac{a_{j}+b_{j}}{2} \quad, \quad i_{j}=\frac{a_{j}-b_{j}}{2 Z_{j j}} .
$$

Though the definition in (1) is the most widespread, other definitions of WD variables with one free parameter per port have been proposed in the literature on WDFs [17], [26][28]. Among them are current waves, or power-normalized waves, which are characterized by different units of measure. A more general definition of biparametric waves, characterized by two free parameters per port instead of just one, was 
introduced in [29], with significant impact on the resulting WDF structures. Such types of WDFs, however, are all based on scalar definitions of waves, as each free parameter refers to a single port of a WD element. When two-port circuit elements are present, a WDF structure based on scalar port-wise definitions of waves is generally affected by computability problems. For example, Fig. 2 shows a WDF structure based on scalar wave variables that includes a generic (linear or nonlinear) two-port element whose ports are both connected to the same topological junction. Dashed paths denote two delayfree-loops that are unavoidably formed in the WDF, which cannot be eliminated through any choice of free parameters.

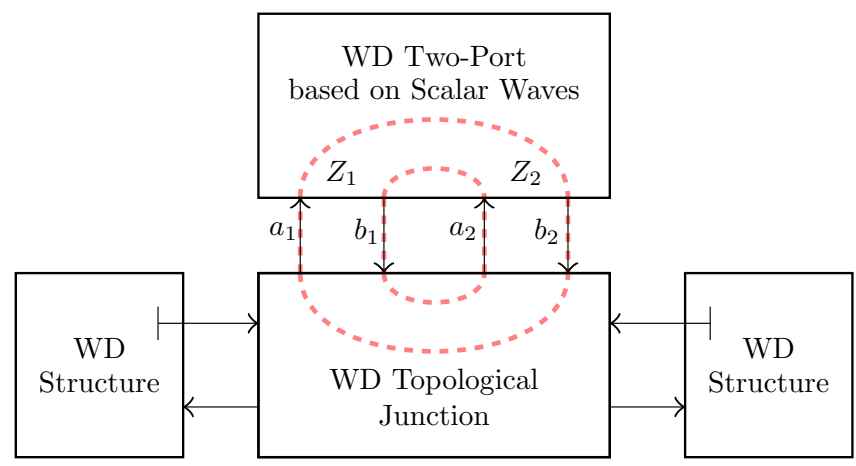

Fig. 2. Example of traditional WDF based on a scalar definition of wave variables. The WDF includes a WD two-port element connected to a WD topological junction. The red dashed circles indicate two delay-free-loops that unavoidably arise in the WDF.

In the following, we re-define wave variables in vector form so as to encompass both ports of the same two-port element. We will see that this will bring advantages in the design of WDFs with two-ports, including in many situations the possibility to remove the delay-free-loops of Fig. 2

\section{B. Vector Definition of Wave Variables with Reference Two- Port Resistance}

With reference to the same generic two-port circuit element shown in Fig. 1, we propose the following vector definition of WD variables, which generalizes (1),

$$
\begin{aligned}
& {\left[\begin{array}{l}
a_{1} \\
a_{2}
\end{array}\right]=\left[\begin{array}{l}
v_{1} \\
v_{2}
\end{array}\right]+\left[\begin{array}{ll}
Z_{11} & Z_{12} \\
Z_{21} & Z_{22}
\end{array}\right]\left[\begin{array}{l}
i_{1} \\
i_{2}
\end{array}\right]} \\
& {\left[\begin{array}{l}
b_{1} \\
b_{2}
\end{array}\right]=\left[\begin{array}{l}
v_{1} \\
v_{2}
\end{array}\right]-\left[\begin{array}{ll}
Z_{11} & Z_{12} \\
Z_{21} & Z_{22}
\end{array}\right]\left[\begin{array}{l}
i_{1} \\
i_{2}
\end{array}\right]}
\end{aligned}
$$

where $\left[v_{1}, v_{2}\right]^{T}$ is the vector of the two port voltages, $\left[i_{1}, i_{2}\right]^{T}$ is the vector of the two port currents, $\left[a_{1}, a_{2}\right]^{T}$ is the vector of the waves incident to the two-port element, $\left[b_{1}, b_{2}\right]^{T}$ is the vector of the waves reflected by the two-port element and

$$
\mathbf{Z}_{1,2}=\left[\begin{array}{ll}
Z_{11} & Z_{12} \\
Z_{21} & Z_{22}
\end{array}\right]
$$

is a full-rank $2 \times 2$ matrix of real free parameters $Z_{k j}$, with $k \in\{1,2\}$ and $j \in\{1,2\}$, which we refer to as reference two-port resistance. As $\mathbf{Z}_{1,2}$ is full-rank, we have

$$
\left|\mathbf{Z}_{1,2}\right|=\operatorname{det}\left[\mathbf{Z}_{1,2}\right]=Z_{11} Z_{22}-Z_{12} Z_{21} \neq 0 .
$$

This allows us to derive the inverse mapping from WD variables to Kirchhoff variables as

$$
\begin{gathered}
{\left[\begin{array}{l}
v_{1} \\
v_{2}
\end{array}\right]=\frac{1}{2}\left(\left[\begin{array}{l}
a_{1} \\
a_{2}
\end{array}\right]+\left[\begin{array}{l}
b_{1} \\
b_{2}
\end{array}\right]\right)} \\
{\left[\begin{array}{l}
i_{1} \\
i_{2}
\end{array}\right]=\frac{1}{2\left|\mathbf{Z}_{1,2}\right|}\left[\begin{array}{rr}
Z_{22} & -Z_{12} \\
-Z_{21} & Z_{11}
\end{array}\right]\left(\left[\begin{array}{l}
a_{1} \\
a_{2}
\end{array}\right]-\left[\begin{array}{l}
b_{1} \\
b_{2}
\end{array}\right]\right)}
\end{gathered}
$$

It is easy to verify that the traditional port-wise definition of waves (1) can be seen as a particular case of (3), in which $Z_{12}=Z_{21}=0$.

In the next Section, we will show how to derive the scattering relation of a generic WD two-port element based on the vector definition of wave variables (3).

\section{A WD Vector Model for Two-Port Elements}

In this manuscript, we consider the large class of linear memoryless two-port circuit elements that can be described by the following model in the Kirchhoff domain

$$
\Psi \xi-\Phi \mu-\delta=0
$$

where $\boldsymbol{\xi}$ and $\boldsymbol{\mu}$ are two-dimensional column vectors collecting port variables; in particular, vector $\boldsymbol{\xi}$ contains two different variables in the set $\left\{v_{1}, v_{2}, i_{1}, i_{2}\right\}$, collecting port voltages and port currents, while vector $\boldsymbol{\mu}$ contains the remaining two variables. $\boldsymbol{\Psi}$ and $\boldsymbol{\Phi}$ are $2 \times 2$ matrices of real coefficients, while $\boldsymbol{\delta}$ is a two-dimensional column vector of real coefficients. 0 is a two-dimensional column vector of zeros.

According to (6) and (7), vector $\boldsymbol{\xi}$ and vector $\boldsymbol{\mu}$ can be expressed as linear combinations of the vectors of wave variables as follows

$$
\begin{gathered}
\boldsymbol{\xi}=\mathbf{K}_{\xi}\left[\begin{array}{l}
a_{1} \\
a_{2}
\end{array}\right]+\mathbf{P}_{\xi}\left[\begin{array}{l}
b_{1} \\
b_{2}
\end{array}\right] \\
\boldsymbol{\mu}=\mathbf{K}_{\mu}\left[\begin{array}{l}
a_{1} \\
a_{2}
\end{array}\right]-\mathbf{P}_{\mu}\left[\begin{array}{l}
b_{1} \\
b_{2}
\end{array}\right]
\end{gathered}
$$

where $2 \times 2$ matrices $\mathbf{K}_{\xi}, \mathbf{P}_{\xi}, \mathbf{K}_{\mu}$ and $\mathbf{P}_{\mu}$ vary according to the Kirchhoff port variables contained in $\boldsymbol{\xi}$ and $\boldsymbol{\mu}$.

Substituting (9) and (10) in (8), we obtain

$$
\boldsymbol{\Psi} \mathbf{K}_{\xi}\left[\begin{array}{l}
a_{1} \\
a_{2}
\end{array}\right]+\boldsymbol{\Psi} \mathbf{P}_{\xi}\left[\begin{array}{l}
b_{1} \\
b_{2}
\end{array}\right]-\boldsymbol{\Phi K}_{\mu}\left[\begin{array}{l}
a_{1} \\
a_{2}
\end{array}\right]+\mathbf{\Phi} \mathbf{P}_{\mu}\left[\begin{array}{l}
b_{1} \\
b_{2}
\end{array}\right]-\boldsymbol{\delta}=\mathbf{0}
$$

or equivalently

$$
\left(\boldsymbol{\Phi} \mathbf{P}_{\mu}+\mathbf{\Psi} \mathbf{P}_{\xi}\right)\left[\begin{array}{l}
b_{1} \\
b_{2}
\end{array}\right]=\left(\boldsymbol{\Phi} \mathbf{K}_{\mu}-\mathbf{\Psi} \mathbf{K}_{\xi}\right)\left[\begin{array}{l}
a_{1} \\
a_{2}
\end{array}\right]+\boldsymbol{\delta} .
$$

Solving (11) for the vector of reflected waves, we get

$$
\left[\begin{array}{l}
b_{1} \\
b_{2}
\end{array}\right]=\mathbf{P}^{-1} \mathbf{K}\left[\begin{array}{l}
a_{1} \\
a_{2}
\end{array}\right]+\mathbf{P}^{-1} \boldsymbol{\delta}
$$

where

$$
\begin{gathered}
\mathbf{P}=\boldsymbol{\Phi} \mathbf{P}_{\mu}+\boldsymbol{\Psi} \mathbf{P}_{\xi} \\
\mathbf{K}=\boldsymbol{\Phi} \mathbf{K}_{\mu}-\mathbf{\Psi} \mathbf{K}_{\xi}
\end{gathered}
$$

The scattering relation in vector form (12) is the WD model of the two-port element based on the proposed vector definition of waves (3). Such a scattering relation in explicit form exists if and only if

$$
\operatorname{det}[\mathbf{P}] \neq 0
$$


Most linear one-ports (e.g., resistors or resistive sources) in traditional WDF theory can be adapted [1]. Adapting a one-port element in the WD domain means eliminating the instantaneous dependency of the reflected wave signal from the incident wave signal. Similarly, in the following, we will refer to an adapted two-port as a two-port WD element for which the following condition holds

$$
\mathbf{K}=\left[\begin{array}{ll}
0 & 0 \\
0 & 0
\end{array}\right] \text {. }
$$

According to (14), condition (16) can also be written as

$$
\Phi \mathbf{K}_{\mu}=\mathbf{\Psi} \mathbf{K}_{\xi} .
$$

It follows that an adapted two-port is characterized by the following simplified scattering relation

$$
\left[\begin{array}{l}
b_{1} \\
b_{2}
\end{array}\right]=\mathbf{P}^{-1} \boldsymbol{\delta}
$$

in which the dependency of the vector of reflected waves $\left[b_{1}, b_{2}\right]^{T}$ from the vector of incident waves $\left[a_{1}, a_{2}\right]^{T}$ is eliminated.

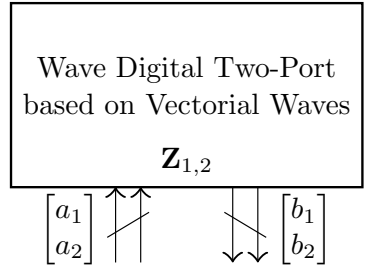

(a)

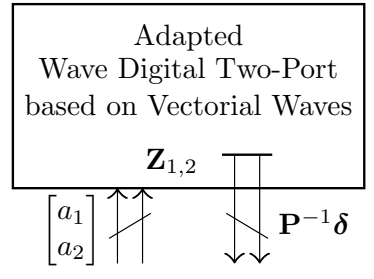

(b)
Fig. 3. Symbol of a WD two-port element based on vector waves (a). Symbol of an adapted WD two-port element based on vector waves (b).

\section{A. Power Absorbed by a WD Two-Port}

Here we provide general considerations about the energetic properties of a WD two-port element based on vector waves in terms of its absorbed power, similarly to what done in reference [2] concerning traditional WDFs. Assuming to deal with real discrete-time signals, the power $W_{1,2}$ absorbed by the two-port shown in Fig. 1 1 can be expressed in the Kirchhoff domain as follows

$$
W_{1,2}=v_{1} i_{1}+v_{2} i_{2} .
$$

Substituting (6) and (7) in (19), we get

$$
W_{1,2}=\frac{1}{4}\left(\left[\begin{array}{ll}
a_{1} & a_{2}
\end{array}\right]+\left[\begin{array}{ll}
b_{1} & b_{2}
\end{array}\right]\right) \mathbf{z}_{1,2}^{-1}\left(\left[\begin{array}{l}
a_{1} \\
a_{2}
\end{array}\right]-\left[\begin{array}{l}
b_{1} \\
b_{2}
\end{array}\right]\right)
$$

or equivalently

$$
\begin{aligned}
W_{1,2}=\frac{1}{4} & \left(\left[\begin{array}{l}
a_{1} \\
a_{2}
\end{array}\right]^{T} \mathbf{Z}_{1,2}^{-1}\left[\begin{array}{l}
a_{1} \\
a_{2}
\end{array}\right]+\left[\begin{array}{l}
b_{1} \\
b_{2}
\end{array}\right]^{T} \mathbf{Z}_{1,2}^{-1}\left[\begin{array}{l}
a_{1} \\
a_{2}
\end{array}\right]+\right. \\
& \left.-\left[\begin{array}{l}
a_{1} \\
a_{2}
\end{array}\right]^{T} \mathbf{Z}_{1,2}^{-1}\left[\begin{array}{l}
b_{1} \\
b_{2}
\end{array}\right]-\left[\begin{array}{l}
b_{1} \\
b_{2}
\end{array}\right]^{T} \mathbf{Z}_{1,2}^{-1}\left[\begin{array}{l}
b_{1} \\
b_{2}
\end{array}\right]\right)
\end{aligned}
$$

which is the same absorbed power in (19), but expressed in the WD domain. The two-port is said passive when $W_{1,2} \geq 0$ and lossless when $W_{1,2}=0$.
We notice that if $\mathbf{Z}_{1,2}^{-1}$ is symmetric, 20) reduces to

$$
W_{1,2}=\frac{1}{4}\left(\left[\begin{array}{l}
a_{1} \\
a_{2}
\end{array}\right]^{T} \mathbf{Z}_{1,2}^{-1}\left[\begin{array}{l}
a_{1} \\
a_{2}
\end{array}\right]-\left[\begin{array}{l}
b_{1} \\
b_{2}
\end{array}\right]^{T} \mathbf{Z}_{1,2}^{-1}\left[\begin{array}{l}
b_{1} \\
b_{2}
\end{array}\right]\right) \text {. }
$$

\section{B. A Special Case: the Subclass of Resistive Two-Ports}

Let us consider the subclass of two-ports such that $\delta=0$ and $\boldsymbol{\Psi}=\mathbf{I}_{2}$, where $\mathbf{I}_{2}$ is the $2 \times 2$ identity matrix. In this case, the scattering relation $(12)$ reduces to

$$
\left[\begin{array}{l}
b_{1} \\
b_{2}
\end{array}\right]=\left(\mathbf{\Phi} \mathbf{P}_{\mu}+\mathbf{P}_{\xi}\right)^{-1}\left(\mathbf{\Phi} \mathbf{K}_{\mu}-\mathbf{K}_{\xi}\right)\left[\begin{array}{l}
a_{1} \\
a_{2}
\end{array}\right],
$$

and, when the adaptation condition (17) is met, we simply have that

$$
\left[\begin{array}{l}
b_{1} \\
b_{2}
\end{array}\right]=\left[\begin{array}{l}
0 \\
0
\end{array}\right] \text {. }
$$

In the next six Sections, we will show that WD models of many important two-ports, namely all the six families of linear resistive two-ports discussed in [25, Chapter 3], can be derived as particular cases of (22).

\section{Current-Controlled Two-Port Elements}

Current-controlled two-port resistive elements in the Kirchhoff domain are characterized by the equation

$$
\left[\begin{array}{l}
v_{1} \\
v_{2}
\end{array}\right]=\left[\begin{array}{ll}
r_{11} & r_{12} \\
r_{21} & r_{22}
\end{array}\right]\left[\begin{array}{l}
i_{1} \\
i_{2}
\end{array}\right]
$$

where $r_{k j}$ are resistance parameters. The corresponding WD model can be derived by taking (22) and setting

$$
\begin{gathered}
\boldsymbol{\xi}=\left[\begin{array}{l}
v_{1} \\
v_{2}
\end{array}\right], \quad \boldsymbol{\mu}=\left[\begin{array}{l}
i_{1} \\
i_{2}
\end{array}\right], \quad \boldsymbol{\Phi}=\mathbf{R}_{1,2}=\left[\begin{array}{ll}
r_{11} & r_{12} \\
r_{21} & r_{22}
\end{array}\right], \\
\mathbf{K}_{\xi}=\mathbf{P}_{\xi}=\frac{1}{2} \mathbf{I}_{2}, \quad \mathbf{K}_{\mu}=\mathbf{P}_{\mu}=\frac{1}{2} \mathbf{Z}_{1,2}^{-1},
\end{gathered}
$$

where $\mathbf{R}_{1,2}$ is the matrix of resistance parameters. The condition (15) on the existence of the scattering relation (22) is satisfied if and only if

$$
\frac{\left|\mathbf{R}_{1,2}\right|+r_{11} Z_{22}-r_{12} Z_{21}-r_{21} Z_{12}+r_{22} Z_{11}+\left|\mathbf{Z}_{1,2}\right|}{4\left|\mathbf{Z}_{1,2}\right|} \neq 0 .
$$

The condition of adaptation (17), that allows us to implement the WD two-port using (23), is satisfied by setting the reference two-port resistance matrix as

$$
\mathbf{Z}_{1,2}=\mathbf{R}_{1,2}
$$

\section{A. Example I: Current-Controlled Voltage Source (CCVS)}

Writing the constitutive equation of the ideal CCVS shown in Fig. 4(a) in terms of resistance parameters, equation (24) reduces to

$$
\left[\begin{array}{l}
v_{1} \\
v_{2}
\end{array}\right]=\left[\begin{array}{cc}
0 & 0 \\
A_{r} & 0
\end{array}\right]\left[\begin{array}{l}
i_{1} \\
i_{2}
\end{array}\right],
$$

where $A_{r}$ is the transresistance gain. Provided that condition 25) is satisfied, the scattering relation of an ideal CCVS is the following

$$
\left[\begin{array}{l}
b_{1} \\
b_{2}
\end{array}\right]=\left[\begin{array}{cc}
-1 & 0 \\
\frac{-2 A_{r} Z_{22}}{A_{r} Z_{12}-\left|\mathbf{Z}_{1,2}\right|} & \frac{A_{r} Z_{12}+\left|\mathbf{Z}_{1,2}\right|}{A_{r} Z_{12}-\left|\mathbf{Z}_{1,2}\right|}
\end{array}\right]\left[\begin{array}{c}
a_{1} \\
a_{2}
\end{array}\right] .
$$


An ideal CCVS cannot be adapted in the WD domain similarly to what happens to one-port ideal voltage sources. However, like the one-port ideal voltage source that can be well approximated by a resistive voltage source with a small series resistance, the ideal CCVS can be well approximated using the transresistance amplifier model described in the next subsection, obtaining an element that can be adapted.

\section{B. Example II: Transresistance Amplifier Model}

The transresistance amplifier model represented in Fig. 4(b) is characterized by the following constitutive equation

$$
\left[\begin{array}{l}
v_{1} \\
v_{2}
\end{array}\right]=\left[\begin{array}{cc}
R_{\text {in }} & 0 \\
A_{r} & R_{\text {out }}
\end{array}\right]\left[\begin{array}{l}
i_{1} \\
i_{2}
\end{array}\right]
$$

where $A_{r}$ is the transresistance gain, $R_{\text {in }}$ is the input resistance and $R_{\text {out }}$ is the output resistance. If resistances $R_{\text {in }}$ and $R_{\text {out }}$ are sufficiently small the model in Fig. 4(b) well approximates the ideal CCVS in Fig. 4(a), The WD realization of the transresistance amplifier model can be adapted and its scattering relation reduces to

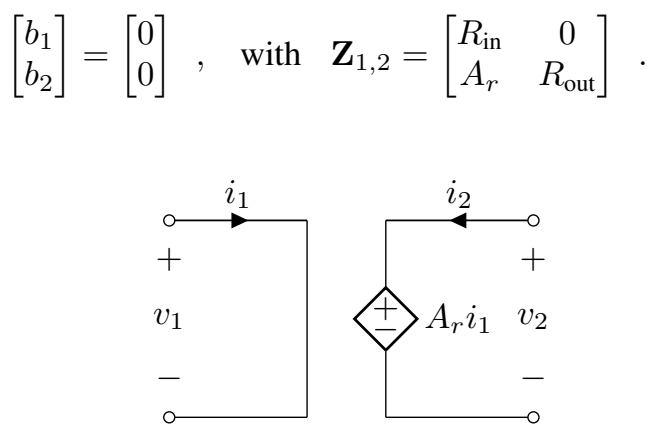

(a)

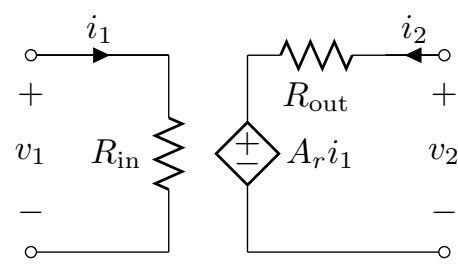

(b)

Fig. 4. Ideal CCVS (a) and transresistance amplifier model (b). If resistances $R_{\text {in }}$ and $R_{\text {out }}$ are sufficiently small the model in (b) well approximates the CCVS in (a).

\section{Voltage-Controlled Two-Port Elements}

Voltage-controlled two-port resistive elements in the Kirchhoff domain are characterized by the equation

$$
\left[\begin{array}{l}
i_{1} \\
i_{2}
\end{array}\right]=\left[\begin{array}{ll}
g_{11} & g_{12} \\
g_{21} & g_{22}
\end{array}\right]\left[\begin{array}{l}
v_{1} \\
v_{2}
\end{array}\right]
$$

where $g_{k j}$ are real conductance parameters. The corresponding WD model can be derived by taking 22, and setting

$$
\begin{gathered}
\boldsymbol{\xi}=\left[\begin{array}{l}
i_{1} \\
i_{2}
\end{array}\right], \quad \boldsymbol{\mu}=\left[\begin{array}{l}
v_{1} \\
v_{2}
\end{array}\right], \quad \boldsymbol{\Phi}=\mathbf{G}_{1,2}=\left[\begin{array}{ll}
g_{11} & g_{12} \\
g_{21} & g_{22}
\end{array}\right], \\
\mathbf{K}_{\xi}=-\mathbf{P}_{\xi}=\frac{1}{2} \mathbf{Z}_{1,2}^{-1}, \quad \mathbf{K}_{\mu}=-\mathbf{P}_{\mu}=\frac{1}{2} \mathbf{I}_{2},
\end{gathered}
$$

where $\mathbf{G}_{1,2}$ is the matrix of conductance parameters. The condition 15 on the existence of the scattering relation 22, is satisfied if and only if

$$
\frac{\left|\mathbf{G}_{1,2}\right|\left|\mathbf{Z}_{1,2}\right|+g_{11} Z_{11}+g_{12} Z_{21}+g_{21} Z_{12}+g_{22} Z_{22}+1}{4\left|\mathbf{Z}_{1,2}\right|} \neq 0 \text {. }
$$

The condition of adaptation (17) is satisfied by setting

$$
\mathbf{Z}_{1,2}=\mathbf{G}_{1,2}^{-1}
$$

provided that $\left|\mathbf{G}_{1,2}\right| \neq 0$.

\section{A. Example I: Voltage-Controlled Current Source (VCCS)}

Writing the constitutive equation of the ideal VCCS shown in Fig. 5(a) in terms of conductance parameters, equation 31 reduces to

$$
\left[\begin{array}{c}
i_{1} \\
i_{2}
\end{array}\right]=\left[\begin{array}{cc}
0 & 0 \\
A_{g} & 0
\end{array}\right]\left[\begin{array}{l}
v_{1} \\
v_{2}
\end{array}\right]
$$

where $A_{g}$ is the transconductance gain. Provided that condition 25] is satisfied, the scattering relation of an ideal VCCS is the following

$$
\left[\begin{array}{l}
b_{1} \\
b_{2}
\end{array}\right]=\left[\begin{array}{ll}
-\frac{A_{g} Z_{12}-1}{A_{g} Z_{12}+1} & 0 \\
-\frac{2 A_{g} Z_{22}}{A_{g} Z_{12}+1} & 1
\end{array}\right]\left[\begin{array}{l}
a_{1} \\
a_{2}
\end{array}\right] .
$$

An ideal VCCS cannot be adapted; however, it can be well approximated using the transconductance amplifier model described in the next subsection that can instead be adapted.

\section{B. Example II: Transconductance Amplifier Model}

The transconductance amplifier model in Fig. 5(b) is characterized by the following constitutive equation

$$
\left[\begin{array}{c}
i_{1} \\
i_{2}
\end{array}\right]=\left[\begin{array}{cc}
1 / R_{\text {in }} & 0 \\
A_{g} & 1 / R_{\text {out }}
\end{array}\right]\left[\begin{array}{l}
v_{1} \\
v_{2}
\end{array}\right]
$$

where $A_{g}$ is the transconductance gain, $R_{\text {in }}$ is the input resistance and $R_{\text {out }}$ is the output resistance. If resistances $R_{\text {in }}$ and $R_{\text {out }}$ are sufficiently large the model in Fig. 5(b) well approximates the ideal VCCS in Fig. 5(a) The WD realization of the transconductance amplifier model can be adapted and its scattering relation reduces to

$$
\left[\begin{array}{l}
b_{1} \\
b_{2}
\end{array}\right]=\left[\begin{array}{l}
0 \\
0
\end{array}\right], \quad \text { with } \quad \mathbf{Z}_{1,2}=\left[\begin{array}{cc}
R_{\text {in }} & 0 \\
-A_{g} R_{\text {in }} R_{\text {out }} & R_{\text {out }}
\end{array}\right] \text {. }
$$

\section{Example III: Gyrator}

The gyrator in Fig. 6 is characterized by the following constitutive equation

$$
\left[\begin{array}{c}
i_{1} \\
i_{2}
\end{array}\right]=\left[\begin{array}{cc}
0 & G_{0} \\
-G_{0} & 0
\end{array}\right]\left[\begin{array}{l}
v_{1} \\
v_{2}
\end{array}\right]
$$

where $G_{0}$ is the gyration conductance. The WD model of the gyrator can be adapted and its scattering relation reduces to

$$
\left[\begin{array}{l}
b_{1} \\
b_{2}
\end{array}\right]=\left[\begin{array}{l}
0 \\
0
\end{array}\right], \text { with } \quad \mathbf{Z}_{1,2}=\left[\begin{array}{cc}
0 & -1 / G_{0} \\
1 / G_{0} & 0
\end{array}\right] .
$$




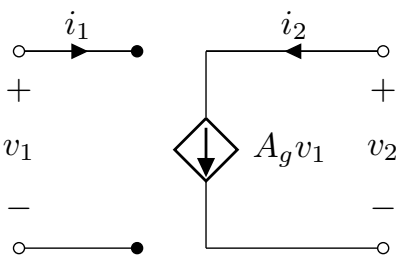

(a)

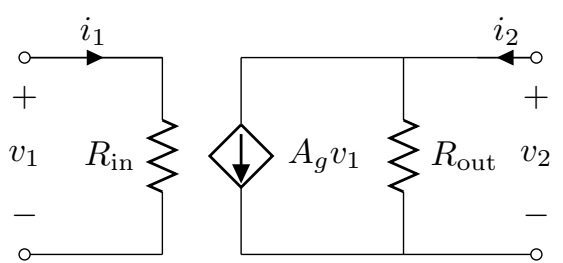

(b)

Fig. 5. Ideal VCCS (a) and transconductance amplifier model (b). If resistances $R_{\text {in }}$ and $R_{\text {out }}$ are sufficiently large the model in (b) well approximates the VCCS in (a).

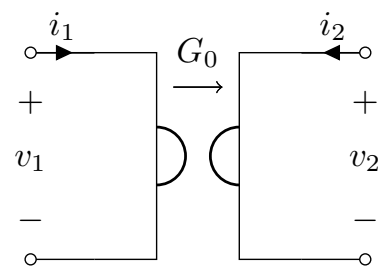

Fig. 6. Gyrator with gyration conductance $G_{0}$.

\section{HybRID Two-PoRT ElEMENTS}

Hybrid two-port resistive elements in the Kirchhoff domain are characterized by the equation

$$
\left[\begin{array}{l}
v_{1} \\
i_{2}
\end{array}\right]=\left[\begin{array}{ll}
h_{11} & h_{12} \\
h_{21} & h_{22}
\end{array}\right]\left[\begin{array}{l}
i_{1} \\
v_{2}
\end{array}\right]
$$

where $h_{k j}$ are real hybrid parameters. The corresponding WD model can be derived by taking (22) and setting

$$
\begin{array}{cc}
\boldsymbol{\xi}=\left[\begin{array}{l}
v_{1} \\
i_{2}
\end{array}\right], \quad \boldsymbol{\mu}=\left[\begin{array}{l}
i_{1} \\
v_{2}
\end{array}\right], \quad \boldsymbol{\Phi}=\mathbf{H}_{1,2}=\left[\begin{array}{cc}
h_{11} & h_{12} \\
h_{21} & h_{22}
\end{array}\right], \\
\mathbf{K}_{\xi}=\frac{1}{2}\left[\begin{array}{cc}
1 & 0 \\
\frac{-Z_{21}}{\left|\mathbf{Z}_{1,2}\right|} & \frac{Z_{11}}{\left|\mathbf{Z}_{1,2}\right|}
\end{array}\right], \quad \mathbf{K}_{\mu}=\frac{1}{2}\left[\begin{array}{cc}
\frac{Z_{22}}{\left|\mathbf{Z}_{1,2}\right|} & \frac{-Z_{12}}{\left|\mathbf{Z}_{1,2}\right|} \\
0 & 1
\end{array}\right], \\
\mathbf{P}_{\xi}=\frac{1}{2}\left[\begin{array}{cc}
1 & 0 \\
\frac{Z_{21}}{\left|\mathbf{Z}_{1,2}\right|} & \frac{-Z_{11}}{\left|\mathbf{Z}_{1,2}\right|}
\end{array}\right], \quad \mathbf{P}_{\mu}=\frac{1}{2}\left[\begin{array}{cc}
\frac{Z_{22}}{\left|\mathbf{Z}_{1,2}\right|} & \frac{-Z_{12}}{\left|\mathbf{Z}_{1,2}\right|} \\
0 & -1
\end{array}\right],
\end{array}
$$

where $\mathbf{H}_{1,2}$ is the matrix of hybrid parameters. The condition (15) on the existence of the scattering relation (22) is satisfied if and only if

$\frac{h_{12} Z_{21}-h_{11}-Z_{11}-h_{21} Z_{12}-Z_{22}\left|\mathbf{H}_{1,2}\right|-h_{22}\left|\mathbf{Z}_{1,2}\right|}{4\left|\mathbf{Z}_{1,2}\right|} \neq 0$.

The condition of adaptation (17) is satisfied by setting

$$
\mathbf{Z}_{1,2}=\frac{1}{h_{22}}\left[\begin{array}{cc}
\left|\mathbf{H}_{1,2}\right| & h_{12} \\
-h_{21} & 1
\end{array}\right],
$$

provided that $h_{22} \neq 0$.

\section{A. Example I: Current-Controlled Current Source (CCCS)}

Writing the constitutive equation of the ideal CCCS shown in Fig. 7(a) in terms of hybrid parameters, equation (40) reduces to

$$
\left[\begin{array}{l}
v_{1} \\
i_{2}
\end{array}\right]=\left[\begin{array}{cc}
0 & 0 \\
A_{0} & 0
\end{array}\right]\left[\begin{array}{l}
i_{1} \\
v_{2}
\end{array}\right]
$$

where $A_{0}$ is a dimensionless gain. Provided that condition (41) is satisfied, the scattering relation of an ideal CCCS is the following

$$
\left[\begin{array}{l}
b_{1} \\
b_{2}
\end{array}\right]=\left[\begin{array}{cc}
-1 & 0 \\
-\frac{2\left(Z_{21}+A_{0} Z_{22}\right)}{Z_{11}+A_{0} Z_{12}} & 1
\end{array}\right]\left[\begin{array}{l}
a_{1} \\
a_{2}
\end{array}\right]
$$

An ideal CCCS cannot be adapted; however, it can be well approximated using the current amplifier model described in the next subsection that can instead be adapted.

\section{B. Example II: Current Amplifier Model}

The current amplifier model in Fig. 7(b) is characterized by the following constitutive equation

$$
\left[\begin{array}{c}
v_{1} \\
i_{2}
\end{array}\right]=\left[\begin{array}{cc}
R_{\text {in }} & 0 \\
A_{0} & 1 / R_{\text {out }}
\end{array}\right]\left[\begin{array}{l}
i_{1} \\
v_{2}
\end{array}\right]
$$

where $A_{0}$ is the dimensionless gain, $R_{\text {in }}$ is the input resistance and $R_{\text {out }}$ is the output resistance. If resistance $R_{\text {in }}$ is sufficiently small and resistance $R_{\text {out }}$ is sufficiently large the model in Fig. 7(b) well approximates the ideal CCCS in Fig. 7(a), The WD realization of the current amplifier model can be adapted and its scattering relation reduces to

$$
\left[\begin{array}{l}
b_{1} \\
b_{2}
\end{array}\right]=\left[\begin{array}{l}
0 \\
0
\end{array}\right], \quad \text { with } \quad \mathbf{Z}_{1,2}=\left[\begin{array}{cc}
R_{\text {in }} & 0 \\
-A_{0} R_{\text {out }} & R_{\text {out }}
\end{array}\right] \text {. }
$$
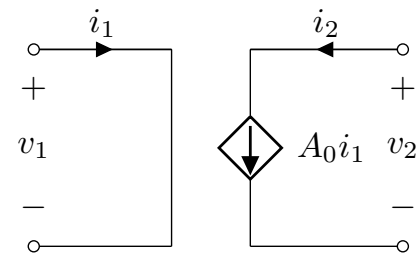

(a)

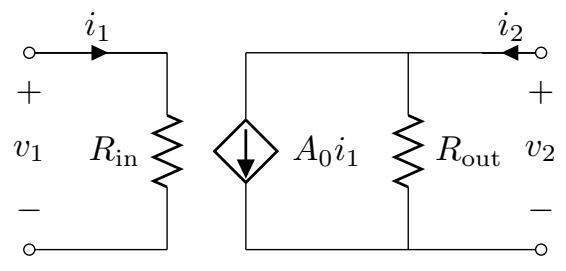

(b)

Fig. 7. Ideal CCCS (a) and current amplifier model (b). If resistance $R_{\text {in }}$ is sufficiently small and resistance $R_{\text {out }}$ is sufficiently large the model in (b) well approximates the CCCS in (a). 


\section{INVERSE HyBRID TWO-PORT ELEMENTS}

Inverse hybrid two-port resistive elements in the Kirchhoff domain are characterized by the equation

$$
\left[\begin{array}{l}
i_{1} \\
v_{2}
\end{array}\right]=\left[\begin{array}{ll}
\tilde{h}_{11} & \tilde{h}_{12} \\
\tilde{h}_{21} & \tilde{h}_{22}
\end{array}\right]\left[\begin{array}{l}
v_{1} \\
i_{2}
\end{array}\right]
$$

where $\tilde{h}_{k j}$ are real inverse hybrid parameters. The corresponding WD model can be derived by taking (22) and setting

$$
\begin{array}{ll}
\boldsymbol{\xi}=\left[\begin{array}{l}
i_{1} \\
v_{2}
\end{array}\right], \quad \boldsymbol{\mu}=\left[\begin{array}{c}
v_{1} \\
i_{2}
\end{array}\right], \quad \boldsymbol{\Phi}=\widetilde{\mathbf{H}}_{1,2}=\left[\begin{array}{cc}
\tilde{h}_{11} & \tilde{h}_{12} \\
\tilde{h}_{21} & \tilde{h}_{22}
\end{array}\right], \\
\mathbf{K}_{\xi}=\frac{1}{2}\left[\begin{array}{cc}
\frac{Z_{22}}{\left|\mathbf{Z}_{1,2}\right|} & \frac{-Z_{12}}{\left|\mathbf{Z}_{1,2}\right|} \\
0 & 1
\end{array}\right], \quad \mathbf{K}_{\mu}=\frac{1}{2}\left[\begin{array}{cc}
1 & 0 \\
\frac{-Z_{21}}{\left|\mathbf{Z}_{1,2}\right|} & \frac{Z_{11}}{\left|\mathbf{Z}_{1,2}\right|}
\end{array}\right], \\
\mathbf{P}_{\xi}=\frac{1}{2}\left[\begin{array}{cc}
\frac{-Z_{22}}{\left|\mathbf{Z}_{1,2}\right|} & \frac{Z_{12}}{\left|\mathbf{Z}_{1,2}\right|} \\
0 & 1
\end{array}\right], \quad \mathbf{P}_{\mu}=\frac{1}{2}\left[\begin{array}{cc}
-1 & 0 \\
\frac{-Z_{21}}{\left|\mathbf{Z}_{1,2}\right|} & \frac{Z_{11}}{\left|\mathbf{Z}_{1,2}\right|}
\end{array}\right],
\end{array}
$$

where $\widetilde{\mathbf{H}}_{1,2}$ is the matrix of inverse hybrid parameters. The condition (15) on the existence of the scattering relation (22) is satisfied if and only if

$$
\frac{\tilde{h}_{21} Z_{12}-\tilde{h}_{22}-Z_{22}-\tilde{h}_{12} Z_{21}-Z_{11}\left|\widetilde{\mathbf{H}}_{1,2}\right|-\tilde{h}_{11}\left|\mathbf{Z}_{1,2}\right|}{4\left|\mathbf{Z}_{1,2}\right|} \neq 0 .
$$

The condition of adaptation (17) is satisfied by setting

$$
\mathbf{Z}_{1,2}=\frac{1}{\tilde{h}_{11}}\left[\begin{array}{cc}
1 & -\tilde{h}_{12} \\
\tilde{h}_{21} & \left|\widetilde{\mathbf{H}}_{1,2}\right|
\end{array}\right]
$$

provided that $\tilde{h}_{11} \neq 0$.

\section{A. Example I: Voltage-Controlled Voltage Source (VCVS)}

Writing the constitutive equation of the ideal VCVS shown in Fig. 8(a) in terms of inverse hybrid parameters, equation (46) reduces to

$$
\left[\begin{array}{l}
i_{1} \\
v_{2}
\end{array}\right]=\left[\begin{array}{cc}
0 & 0 \\
A_{0} & 0
\end{array}\right]\left[\begin{array}{l}
v_{1} \\
i_{2}
\end{array}\right]
$$

where $A_{0}$ is a dimensionless gain. Provided that condition (47) is satisfied, the scattering relation of an ideal VCVS is the following

$$
\left[\begin{array}{l}
b_{1} \\
b_{2}
\end{array}\right]=\left[\begin{array}{cc}
\frac{Z_{22}+A_{0} Z_{12}}{Z_{22}-A_{0} Z_{12}} & \frac{-2 Z_{12}}{Z_{22}-A_{0} Z_{12}} \\
\frac{2 A_{0} Z_{22}}{Z_{22}-A_{0} Z_{12}} & \frac{-Z_{22}-A_{0} Z_{12}}{Z_{22}-A_{0} Z_{12}}
\end{array}\right]\left[\begin{array}{l}
a_{1} \\
a_{2}
\end{array}\right] .
$$

\section{B. Example II: Voltage Amplifier Model}

The voltage amplifier model in Fig. $8(\mathrm{~b})$ is characterized by the following constitutive equation

$$
\left[\begin{array}{l}
i_{1} \\
v_{2}
\end{array}\right]=\left[\begin{array}{cc}
1 / R_{\text {in }} & 0 \\
A_{0} & R_{\text {out }}
\end{array}\right]\left[\begin{array}{l}
v_{1} \\
i_{2}
\end{array}\right]
$$

where $A_{0}$ is the dimensionless gain, $R_{\text {in }}$ is the input resistance and $R_{\text {out }}$ is the output resistance. If $R_{\text {in }}$ is sufficiently large and $R_{\text {out }}$ is sufficiently small the model in Fig. 8(b) well approximates the ideal VCVS in Fig. 8(a) The WD realization of the voltage amplifier model can be adapted and its scattering relation reduces to

$$
\left[\begin{array}{l}
b_{1} \\
b_{2}
\end{array}\right]=\left[\begin{array}{l}
0 \\
0
\end{array}\right] \text {, with } \quad \mathbf{Z}_{1,2}=\left[\begin{array}{cc}
R_{\text {in }} & 0 \\
A_{0} R_{\text {in }} & R_{\text {out }}
\end{array}\right] \text {. }
$$

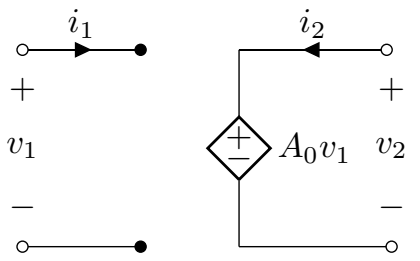

(a)

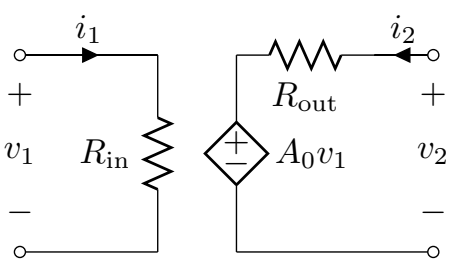

(b)

Fig. 8. Ideal VCVS (a) and voltage amplifier model (b). If $R_{\text {in }}$ is sufficiently large and $R_{\text {out }}$ is sufficiently small the model in (b) well approximates the VCVS in (a).

\section{TRANSMISSION TWO-PORT ELEMENTS}

Transmission two-port resistive elements in the Kirchhoff domain are characterized by the equation

$$
\left[\begin{array}{l}
v_{1} \\
i_{1}
\end{array}\right]=\left[\begin{array}{ll}
t_{11} & t_{12} \\
t_{21} & t_{22}
\end{array}\right]\left[\begin{array}{r}
v_{2} \\
-i_{2}
\end{array}\right]
$$

where $t_{k j}$ are real transmission parameters, also known as ABCD parameters. The corresponding WD model can be derived by taking (22) and setting

$$
\begin{array}{ll}
\boldsymbol{\xi}=\left[\begin{array}{l}
v_{1} \\
i_{1}
\end{array}\right], \quad \boldsymbol{\mu}=\left[\begin{array}{r}
v_{2} \\
-i_{2}
\end{array}\right], \quad \boldsymbol{\Phi}=\mathbf{T}_{1,2}=\left[\begin{array}{ll}
t_{11} & t_{12} \\
t_{21} & t_{22}
\end{array}\right], \\
\mathbf{K}_{\xi}=\frac{1}{2}\left[\begin{array}{cc}
1 & 0 \\
\frac{Z_{22}}{\left|\mathbf{Z}_{1,2}\right|} & \frac{-Z_{12}}{\left|\mathbf{Z}_{1,2}\right|}
\end{array}\right], \quad \mathbf{K}_{\mu}=\frac{1}{2}\left[\begin{array}{cc}
0 & 1 \\
\frac{Z_{21}}{\left|\mathbf{Z}_{1,2}\right|} & \frac{-Z_{11}}{\left|\mathbf{Z}_{1,2}\right|}
\end{array}\right], \\
\mathbf{P}_{\xi}=\frac{1}{2}\left[\begin{array}{cc}
1 & 0 \\
\frac{-Z_{22}}{\left|\mathbf{Z}_{1,2}\right|} & \frac{Z_{12}}{\left|\mathbf{Z}_{1,2}\right|}
\end{array}\right], \quad \mathbf{P}_{\mu}=\frac{1}{2}\left[\begin{array}{cc}
0 & -1 \\
\frac{Z_{21}}{\left|\mathbf{Z}_{1,2}\right|} & \frac{-Z_{11}}{\left|\mathbf{Z}_{1,2}\right|}
\end{array}\right],
\end{array}
$$

where $\mathbf{T}_{1,2}$ is the matrix of transmission parameters. The condition (15) on the existence of the scattering relation 22] is satisfied if and only if

$\frac{Z_{12}-t_{12}-t_{11} Z_{22}-t_{22} Z_{11}+Z_{21}\left|\mathbf{T}_{1,2}\right|-t_{21}\left|\mathbf{Z}_{1,2}\right|}{4\left|\mathbf{Z}_{1,2}\right|} \neq 0$.

The condition of adaptation (17) is satisfied by setting

$$
\mathbf{Z}_{1,2}=\frac{1}{t_{21}}\left[\begin{array}{cc}
t_{11} & \left|\mathbf{T}_{1,2}\right| \\
1 & t_{22}
\end{array}\right],
$$

provided that $t_{21} \neq 0$.

\section{A. Example: Nullor}

The two-port nullor in Fig. 9 is composed of a one-port nullator (at the left) and a one-port norator (at the right) [24]. It is characterized by the following constitutive equation

$$
\left[\begin{array}{l}
v_{1} \\
i_{1}
\end{array}\right]=\left[\begin{array}{ll}
0 & 0 \\
0 & 0
\end{array}\right]\left[\begin{array}{r}
v_{2} \\
-i_{2}
\end{array}\right] .
$$

A two-port WD realization of a nullor based on a scalar definition of wave variables is not feasible as already discussed 
in [17], [24]. However, a two-port WD realization of the nullor based on vectorial waves can be derived. It can be verified that the condition of existence of the scattering relation 53. reduces to

$$
Z_{12} \neq 0 \wedge\left|\mathbf{Z}_{1,2}\right| \neq 0
$$

It follows that, setting (55), the scattering relation of a WD nullor is given by

$$
\left[\begin{array}{l}
b_{1} \\
b_{2}
\end{array}\right]=\left[\begin{array}{cc}
-1 & 0 \\
-\frac{2 Z_{22}}{Z_{12}} & 1
\end{array}\right]\left[\begin{array}{l}
a_{1} \\
a_{2}
\end{array}\right]
$$

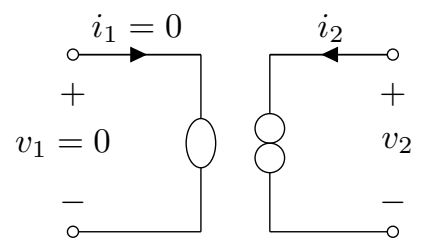

Fig. 9. Two-port nullor composed of a nullator and a norator.

It is worth noticing that a two-port WD nullor cannot be adapted.

\section{INVERSE TRANSMission Two-Port ElEMENTS}

Inverse transmission two-port resistive elements in the Kirchhoff domain are characterized by the equation

$$
\left[\begin{array}{r}
v_{2} \\
-i_{2}
\end{array}\right]=\left[\begin{array}{ll}
\tilde{t}_{11} & \tilde{t}_{12} \\
\tilde{t}_{21} & \tilde{t}_{22}
\end{array}\right]\left[\begin{array}{l}
v_{1} \\
i_{1}
\end{array}\right]
$$

where $\tilde{t}_{k j}$ are real inverse transmission parameters. The corresponding WD model can be derived by taking (22) and setting

$$
\begin{aligned}
& \boldsymbol{\xi}=\left[\begin{array}{r}
v_{2} \\
-i_{2}
\end{array}\right], \quad \boldsymbol{\mu}=\left[\begin{array}{l}
v_{1} \\
i_{1}
\end{array}\right], \quad \boldsymbol{\Phi}=\widetilde{\mathbf{T}}_{1,2}=\left[\begin{array}{cc}
\tilde{t}_{11} & \tilde{t}_{12} \\
\tilde{t}_{21} & \tilde{t}_{22}
\end{array}\right], \\
& \mathbf{K}_{\xi}=\frac{1}{2}\left[\begin{array}{cc}
0 & 1 \\
\frac{Z_{21}}{\left|\mathbf{Z}_{1,2}\right|} & \frac{-Z_{11}}{\left|\mathbf{Z}_{1,2}\right|}
\end{array}\right], \quad \mathbf{K}_{\mu}=\frac{1}{2}\left[\begin{array}{cc}
1 & 0 \\
\frac{Z_{22}}{\left|\mathbf{Z}_{1,2}\right|} & \frac{-Z_{12}}{\left|\mathbf{Z}_{1,2}\right|}
\end{array}\right], \\
& \mathbf{P}_{\xi}=\frac{1}{2}\left[\begin{array}{cc}
0 & 1 \\
\frac{-Z_{21}}{\left|\mathbf{Z}_{1,2}\right|} & \frac{Z_{11}}{\left|\mathbf{Z}_{1,2}\right|}
\end{array}\right], \quad \mathbf{P}_{\mu}=\frac{1}{2}\left[\begin{array}{cc}
-1 & 0 \\
\frac{Z_{22}}{\left|\mathbf{Z}_{1,2}\right|} & \frac{-Z_{12}}{\left|\mathbf{Z}_{1,2}\right|}
\end{array}\right],
\end{aligned}
$$

where $\widetilde{\mathbf{T}}_{1,2}$ is the matrix of inverse transmission parameters. The condition (15) on the existence of the scattering relation (22) is satisfied if and only if

$$
\frac{Z_{21}+\tilde{t}_{12}-\tilde{t}_{11} Z_{11}-\tilde{t}_{22} Z_{22}+Z_{12}\left|\widetilde{\mathbf{T}}_{1,2}\right|+\tilde{t}_{21}\left|\mathbf{Z}_{1,2}\right|}{4\left|\mathbf{Z}_{1,2}\right|} \neq 0 .
$$

The condition of adaptation (17) is satisfied by setting

$$
\mathbf{Z}_{1,2}=\frac{-1}{\tilde{t}_{21}}\left[\begin{array}{cc}
\tilde{t}_{22} & 1 \\
\left|\widetilde{\mathbf{T}}_{1,2}\right| & \tilde{t}_{11}
\end{array}\right] \text {, }
$$

provided that $\tilde{t}_{21} \neq 0$.

\section{Modeling Topological Junctions Based on} Mixed Scalar and Vector Definitions of WaVES

Two main different approaches for modeling topological junctions and, more generally, multi-port connection networks are available in the literature on traditional WDFs based on scalar wave variables. The former consists of connecting instantaneous Thévenin or Norton equivalents to the ports of the target connection network in the Kirchhoff domain and then solving the resulting circuit using the Modified Nodal Analysis [17], [20]. This method can be used for deriving the scattering matrix of whatever reciprocal or nonreciprocal connection network. The latter approach was developed for modeling reciprocal connection networks using different definitions of wave variables [15], [16], [18]. This is based on the derivation of a fundamental cut-set matrix (or a dual fundamental loop matrix) that characterizes the relations between Kirchhoff port variables. In this case, the scattering matrix of the junction can be obtained in closed-form as a function of the fundamental cut-set matrix (or the fundamental loop matrix). Though less general, as it only works with reciprocal connection networks, the latter approach [18] surpasses (or at least matches) the performance of the former [17], as far as the cost of the formation of the scattering matrix and the computation of the waves reflected from the junction are concerned. It is worth recalling that purely topological junctions (i.e., wire interconnections) are all intrinsically reciprocal [18], [30].

As mentioned in the Introduction, in many scenarios, the computability problems arising in WDFs with linear two-ports can be circumvented by embedding the two-ports in a topological multi-port junction. However, this modeling strategy partially undermines the modularity property of traditional WDFs formalized by Fettweis [1], according to which topological junctions (adaptors) and circuit elements are implemented in the WD domain using separated input-output blocks. Moreover, as a further side effect, when the embedded two-ports are non-reciprocal also the resulting connection network might be non-reciprocal and the efficient modeling method described in [15], [16], [18] cannot be used for the realization of the corresponding WD junction.

This Section provides a generalization of the approach presented in [15], [16], [18] which allows us to design topological multi-port WD junctions based on mixed scalar and vector definitions of waves. The resulting WD junctions can be used to interconnect one-ports based on traditional scalar waves and the two-ports based on vector waves, thus solving many of the computability problems that would arise using scalar definitions, while preserving modularity, i.e., modeling circuit elements and topology in a separate fashion.

Let us consider a $N$-port topological junction that is connected to other blocks (elements or other junctions) through $P$ two-port connections and $N-2 P$ one-port connections. We collect port voltages in vector $\mathbf{v}_{\mathbf{J}}=\left[v_{\mathrm{J} 1}, \ldots, v_{\mathbf{J} N}\right]^{T}$ and port currents in vector $\mathbf{i}_{\mathrm{J}}=\left[i_{\mathrm{J} 1}, \ldots, i_{\mathrm{J} N}\right]^{T}$. Since purely topological junctions are reciprocal, we can always write [18]

$$
\mathbf{v}_{\mathbf{J}}=\mathbf{Q}^{T} \mathbf{v}_{\mathrm{Jt}}, \quad \mathbf{i}_{\mathrm{J}}=\mathbf{B}^{T} \mathbf{i}_{\mathrm{Jl}},
$$

where $\mathbf{v}_{\mathrm{Jt}}$ is a column vector of size $q, 1 \leq q<N$, containing independent port voltages and $\mathbf{i}_{\mathrm{Jl}}$ is a column vector of size 
$N-q$ containing independent port currents. $\mathbf{Q}$ and $\mathbf{B}$ are the $q \times N$ fundamental cut-set matrix and the $N-q \times N$ fundamental loop matrix, respectively, and they satisfy the orthogonality property $\mathbf{B Q}^{T}=\mathbf{0}$, where $\mathbf{0}$ is a zero matrix of proper size. Kirchhoff port variables are mapped to wave variables according to

$$
\mathbf{v}_{\mathrm{J}}=\frac{1}{2}\left(\mathbf{a}_{\mathrm{J}}+\mathbf{b}_{\mathrm{J}}\right) \quad, \quad \mathbf{i}_{\mathrm{J}}=\frac{1}{2} \mathbf{Z}_{\mathrm{J}}^{-1}\left(\mathbf{a}_{\mathrm{J}}-\mathbf{b}_{\mathrm{J}}\right),
$$

where $\mathbf{a}_{\mathbf{J}}=\left[a_{\mathrm{J} 1}, \ldots, a_{\mathrm{J} N}\right]^{T}$ is the vector of waves entering the junction, $\mathbf{b}_{\mathrm{J}}=\left[b_{\mathrm{J} 1}, \ldots, b_{\mathrm{J} N}\right]^{T}$ is the vector of waves reflected by the junction, while $\mathbf{Z}_{\mathrm{J}}$ is a full-rank block-diagonal matrix of free parameters (not simply a diagonal matrix as in traditional WDFs based on scalar waves). Assuming, without loss of generality, to number the $P$ two-port connections before the $N-2 P$ one-port connections, matrix $\mathbf{Z}_{\mathrm{J}}$ can be written as

$\mathbf{Z}_{\mathbf{J}}=\left[\begin{array}{ccccccc}\mathbf{Z}_{1,2} & \mathbf{0} & \ldots & \mathbf{0} & {[0,0]^{T}} & \ldots & {[0,0]^{T}} \\ \mathbf{0} & \mathbf{Z}_{3,4} & \ldots & \mathbf{0} & {[0,0]^{T}} & \ldots & {[0,0]^{T}} \\ \vdots & \vdots & \ddots & \vdots & \vdots & \ddots & \vdots \\ \mathbf{0} & \mathbf{0} & \ldots & \mathbf{Z}_{2 P-1,2 P} & {[0,0]^{T}} & \ldots & {[0,0]^{T}} \\ {[0,0]} & {[0,0]} & \ldots & {[0,0]} & Z_{2 P+1} & \ldots & 0 \\ \vdots & \vdots & \ddots & \vdots & \vdots & \ddots & \vdots \\ {[0,0]} & {[0,0]} & \ldots & {[0,0]} & 0 & \ldots & Z_{N}\end{array}\right]$

where $\mathbf{Z}_{1,2}, \ldots, \mathbf{Z}_{2 P-1,2 P}$ are $2 \times 2$ full-rank submatrices and $Z_{2 P+1}, \ldots, Z_{N}$ are scalar parameters different from zero.

For example, let us assume that a generic two-port, such as the one described in Subsection II-B, is connected to ports 1 and 2 of the junction. It follows that

$$
v_{\mathrm{J} 1}=v_{1}, \quad v_{\mathrm{J} 2}=v_{2}, \quad i_{\mathrm{J} 1}=-i_{1}, \quad i_{\mathrm{J} 2}=-i_{2},
$$

where $v_{1}, v_{2}, i_{1}$ and $i_{2}$ are the Kirchhoff port variables of the two-port element. Eq. (61) and eq. 62 also imply the following constraints in the WD domain

$$
a_{\mathrm{J} 1}=b_{1} \quad, \quad a_{\mathrm{J} 2}=b_{2} \quad, \quad b_{\mathrm{J} 1}=a_{1}, \quad b_{\mathrm{J} 2}=a_{2},
$$

where $a_{1}, a_{2}, b_{1}$ and $b_{2}$ are the wave variables of the two-port element. Moreover, the $2 \times 2$ submatrix $\mathbf{Z}_{1,2}$ of $\mathbf{Z}_{\mathrm{J}}$ is set equal to the reference two-port resistance matrix of eq. (4).

Waves reflected by the junction $b_{\mathrm{J}}$ and waves incident to junction $\mathrm{a}_{\mathrm{J}}$ are related by the scattering relation

$$
\mathbf{b}_{\mathrm{J}}=\mathbf{S} \mathbf{a}_{\mathrm{J}} \text {. }
$$

Two closed-form expressions of $\mathbf{S}$ are obtained starting from eq. (60) and eq. [59, similarly to what done in [15] and in [18], but in the more general case in which $\mathbf{Z}_{\mathrm{J}}$ is fullrank block-diagonal instead of full-rank diagonal. The two equivalent formulas for computing $\mathbf{S}$ are

$$
\begin{aligned}
& \mathbf{S}=2 \mathbf{Q}^{T}\left(\mathbf{Q Z}_{\mathbf{J}}^{-1} \mathbf{Q}^{T}\right)^{-1} \mathbf{Q Z}_{\mathrm{J}}^{-1}-\mathbf{I}_{N}, \\
& \mathbf{S}=\mathbf{I}_{N}-2 \mathbf{Z}_{\mathbf{J}} \mathbf{B}^{T}\left(\mathbf{B} \mathbf{Z}_{\mathbf{J}} \mathbf{B}^{T}\right)^{-1} \mathbf{B},
\end{aligned}
$$

where $\mathbf{I}_{N}$ is the $N \times N$ identity matrix.

In the following subsections we discuss two fundamental properties of the scattering matrix $\mathbf{S}$ and, finally, we show how to derive it in a practical example.

\section{A. Losslessness Property}

Topological connection networks are lossless, therefore

$$
W_{\mathbf{J}}=\mathbf{v}_{\mathbf{J}}^{T} \mathbf{i}_{\mathbf{J}}=0
$$

According to 67, in the WD domain we can write

$$
W_{\mathbf{J}}=\frac{1}{4}\left(\mathbf{a}_{\mathbf{J}}+\mathbf{S} \mathbf{a}_{\mathbf{J}}\right)^{T} \mathbf{Z}_{\mathbf{J}}^{-1}\left(\mathbf{a}_{\mathbf{J}}-\mathbf{S} \mathbf{a}_{\mathbf{J}}\right),
$$

or equivalently

$$
W_{\mathrm{J}}=\frac{1}{4} \mathbf{a}_{\mathrm{J}}^{T}\left(\mathbf{I}_{N}+\mathbf{S}^{T}\right) \mathbf{Z}_{\mathrm{J}}^{-1}\left(\mathbf{I}_{N}-\mathbf{S}\right) \mathbf{a}_{\mathbf{J}} .
$$

It follows that, for arbitrary wave signals in $\mathbf{a}_{\mathrm{J}}$, losslessness implies the condition

$$
\left(\mathbf{I}_{N}+\mathbf{S}^{T}\right) \mathbf{Z}_{\mathrm{J}}^{-1}\left(\mathbf{I}_{N}-\mathbf{S}\right)=\mathbf{0} .
$$

If $\mathbf{Z}_{\mathrm{J}}$ is symmetric (which is always the case in traditional WDFs, but it is not in WDFs based on vectorial waves) does eq. (68) reduce to

$$
W_{\mathbf{J}}=\frac{1}{4}\left(\mathbf{a}_{\mathbf{J}}^{T} \mathbf{Z}_{\mathbf{J}}^{-1} \mathbf{a}_{\mathbf{J}}-\mathbf{b}_{\mathbf{J}}^{T} \mathbf{Z}_{\mathbf{J}}^{-1} \mathbf{b}_{\mathbf{J}}\right)
$$

or equivalently

$$
W_{\mathbf{J}}=\frac{1}{4} \mathbf{a}_{\mathbf{J}}^{T}\left(\mathbf{Z}_{\mathbf{J}}^{-1}-\mathbf{S}^{T} \mathbf{Z}_{\mathbf{J}}^{-1} \mathbf{S}\right) \mathbf{a}_{\mathbf{J}} .
$$

Hence, if $\mathbf{Z}_{\mathrm{J}}$ is symmetric, does the losslessness condition 70 simplify to [18]

$$
\mathbf{S}^{T} \mathbf{Z}_{\mathrm{J}}^{-1} \mathbf{S}=\mathbf{Z}_{\mathrm{J}}^{-1}
$$

\section{B. Self-inverse Property}

Similarly to what pointed out in [31], since $\mathbf{v}_{\mathbf{J}}$ and $\mathbf{i}_{\mathbf{J}}$ are solutions of independent homogeneous linear equations, we can rewrite the relation (64), i.e., $\mathbf{b}_{\mathrm{J}}=\mathbf{S} \mathbf{a}_{\mathrm{J}}$, as

$$
\xi \mathbf{v}_{\mathbf{J}}-\zeta \mathbf{Z}_{\mathbf{J}} \mathbf{i}_{\mathbf{J}}=\mathbf{S}\left(\xi \mathbf{v}_{\mathbf{J}}+\zeta \mathbf{Z}_{\mathbf{J}} \mathbf{i}_{\mathbf{J}}\right)
$$

where $\xi$ and $\zeta$ are arbitrary real numbers. Hence, choosing $\xi=-1$ and $\zeta=1$, the inverse mapping $\mathbf{a}_{\mathbf{J}}=\mathbf{S} \mathbf{b}_{\mathrm{J}}$ holds true as well. This means that $\mathbf{S}$ is an involutory matrix and the following self-inverse property is satisfied

$$
\mathbf{S S}=\mathbf{I}_{N} .
$$

\section{Case Study}

Let us consider the active band-pass filter with one opamp in Fig. 10(a), The topological connections of the circuit are represented by the directed graph in Fig. 10(b). Each branch of the graph is numbered and it corresponds to one specific port of the elements of the circuit. This means that each one-port element is associated to one branch, while the two-port opamp is associated to two branches of the graph, i.e., branches 1 and 2. Directions of arrows in branches are determined by the chosen polarity of the corresponding port currents. We design a WD model of the reference circuit based on a single 7-port topological junction (called $\mathcal{J}_{1}$ ) to which all the elements are connected, as shown in Fig. 11(a) (further details of such a 


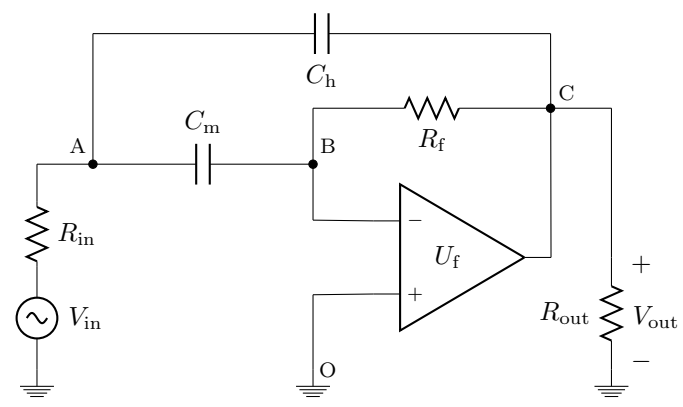

(a)

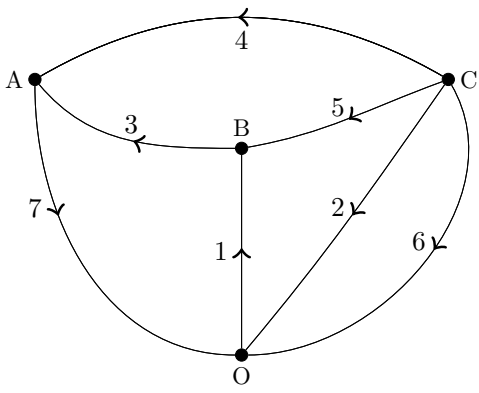

(b)

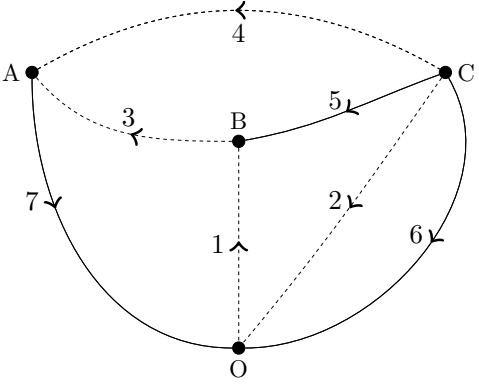

(c)

Fig. 10. Active band-pass filter circuit (a). Directive graph representing the topological connection network to which all the one-port elements and the two-port opamp of the circuit are connected (b). Branches numbered 1 and 2 correspond to the two ports to which the opamp $U_{\mathrm{f}}$ is connected. Decomposition of the directive graph in tree subgraph (continuous branches) and cotree subgraph (dashed branches) (c).

model are provided in the next section). It follows that the matrix of free parameters of the junction is given by

$$
\mathbf{Z}_{\mathbf{J}}=\left[\begin{array}{ccccccc}
Z_{1,1} & Z_{1,2} & 0 & 0 & 0 & 0 & 0 \\
Z_{2,1} & Z_{2,2} & 0 & 0 & 0 & 0 & 0 \\
0 & 0 & Z_{3} & 0 & 0 & 0 & 0 \\
0 & 0 & 0 & Z_{4} & 0 & 0 & 0 \\
0 & 0 & 0 & 0 & Z_{5} & 0 & 0 \\
0 & 0 & 0 & 0 & 0 & Z_{6} & 0 \\
0 & 0 & 0 & 0 & 0 & 0 & Z_{7}
\end{array}\right] .
$$

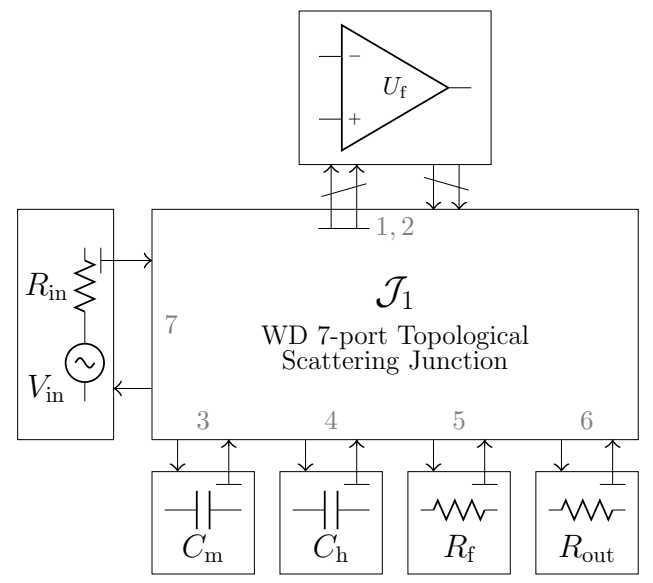

(a)

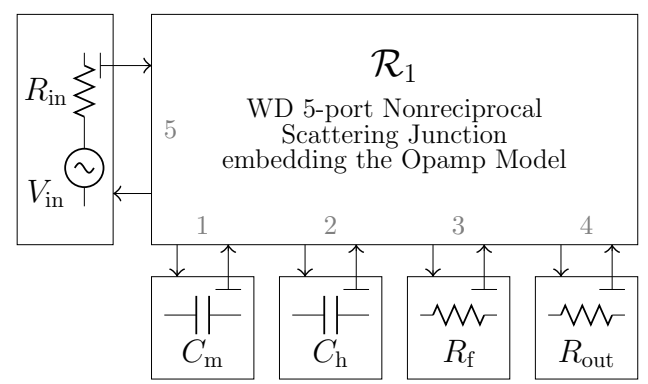

(b)

Fig. 11. WDF realizations of the circuit in Fig. 10(a)

In order to derive the scattering matrix of the junction $\mathbf{S}_{\mathcal{J}_{1}}$ we perform a tree-cotree decomposition that allows us to easily identify the subset of independent port voltages or independent port currents, as explained in [18]. A tree-cotree partition is represented in Fig. 10(c), where the tree subgraph has continuous branches (called twigs), while the cotree subgraph has dashed branches (called links). Since twigs are less than links (3 vs 4 ), eq. (65) is the most computationally efficient formula to derive the scattering matrix [15], [18]. Independent port voltages, related to the 3 twigs of the tree, are collected in the vector $\mathbf{v}_{\mathrm{Jt}}=\left[v_{5}, v_{6}, v_{7}\right]^{T}$ and, according to eq. (59), the fundamental cut-set matrix is expressed as

$$
\mathbf{Q}=\left[\begin{array}{rrrrrrr}
1 & 0 & -1 & 0 & 1 & 0 & 0 \\
-1 & 1 & 1 & 1 & 0 & 1 & 0 \\
0 & 0 & -1 & -1 & 0 & 0 & 1
\end{array}\right]
$$

\section{EXAMPLES OF APPLICATIONS}

This Section discusses how two circuits with two-port elements can be implemented in the WD domain using the vector waves introduced in this manuscript. Comparisons between the resulting WD structures and state-of-the-art structures [17] are also provided, highlighting the benefits of the proposed approach. The two-ports considered in this Section are all 3terminal opamps. Opamps can be implemented using various models characterized by a different "degree of ideality". The models that we refer to are summarized in Fig. 12 .

The modeling of linear one-ports is based on traditional WDF principles [1]; in particular, the trapezoidal rule is used for discretizing the time derivatives present in the constitutive equations of dynamic elements. Each linear one-port that admits an adaptation condition is adapted, i.e., the local instantaneous dependency between the wave incident to the element and the wave reflected by the element is eliminated by properly setting the reference one-port resistance [1].

All the discussed WD implementations are performed in the discrete-time domain using a sampling frequency $F_{\mathrm{s}}=96$ $\mathrm{kHz}$. The frequency responses are obtaining computing the Discrete Fourier Transform of the time-domain impulse responses. In both the examples, the input signal is the voltage source $V_{\text {in }}$, while the output signal is the voltage $V_{\text {out }}$ across the resistor $R_{\text {out }}$. 


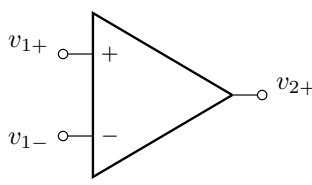

(a)

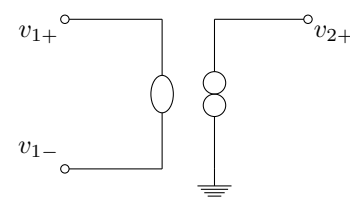

(b)

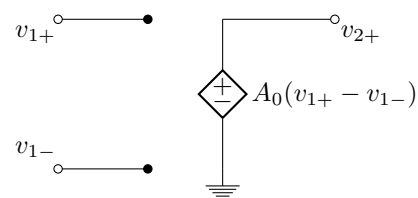

(c)

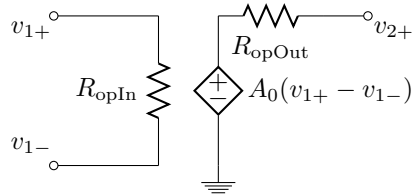

(d)

Fig. 12. Symbol of opamp with 3 terminals (a). $v_{1+}, v_{1-}$ and $v_{2+}$ are the potentials at the 3 terminals. Port voltages are defined as $v_{1}=v_{1+}-v_{1-}$ and $v_{2}=v_{2}$. Opamp model based on nullor (b). Opamp model based on ideal VCVS (c). Opamp model based on VCVS, input resistor and output resistor (d).

\section{A. WD Implementations of the Active Band-Pass Filter Circuit with One Opamp}

The active band-pass filter circuit in Fig. 10(a) is characterized by the following parameters: $R_{\mathrm{in}}=10 \mathrm{k} \Omega, R_{\mathrm{f}}=20 \mathrm{k} \Omega$, $R_{\text {out }}=100 \mathrm{k} \Omega$ and $C_{\mathrm{m}}=C_{\mathrm{h}}=11.2 \mathrm{nF}$. For now we assume that the opamp $U_{\mathrm{f}}$ is described using the ideal nullor-based model in Fig. 12(b) Let us consider the two different WD implementations in Fig. 11.

The WD structure in Fig. 11(a), based on the vector definition of waves at the ports of the opamp is characterized by the 7-port WD topological junction $\mathcal{J}_{1}$ already discussed in Subsection $\mathrm{X}-\mathrm{C}$. The scattering matrix $\mathbf{S}_{\mathcal{J}_{1}}$ is computed substituting the $3 \times 7$ matrix $\mathbf{Q}$ in (77) and the $7 \times 7$ matrix $\mathbf{Z}_{\mathrm{J}}$ in (76) into eq. 65). The nullor-based model of the opamp $U_{\mathrm{f}}$ is implemented using equation (56). It is worth remarking the fact that the proposed definition of waves allows us, for the first time in the literature, to treat the nullor as a two-port WD element characterized by its own scattering relation. The free parameters $Z_{1,1}, Z_{1,2}, Z_{2,1}$ and $Z_{2,2}$ are set in such a way that the four entries of the first $2 \times 2$ diagonal submatrix of $\mathbf{S}_{\mathcal{J}_{1}}$ are zeroed, hence the opamp does not create any delay-free-loop despite it is connected to the junction through a double port connection. Adaptation conditions on the free parameters are not included here for reasons of space, but they can be easily derived using a symbolic math software.

The WD structure in Fig. 11(b) instead, is designed following the approach in [17] based on traditional scalar port-wise definitions of waves. For computability reasons the nullor is embedded in the connection network, which is modeled in the WD domain using a 5-port non-reciprocal junction $\mathcal{R}_{1}$. The scattering matrix of $\mathcal{R}_{1}$ is computed as

$$
\mathbf{S}_{\mathcal{R}_{1}}=2 \widetilde{\mathbf{A}}_{\mathrm{p}}^{T}\left[\begin{array}{ll}
\mathbf{I}_{3} & \mathbf{0}
\end{array}\right] \widetilde{\mathbf{X}}_{0}^{-1}\left[\begin{array}{ll}
\mathbf{I}_{3} & \mathbf{0}
\end{array}\right]^{T} \widetilde{\mathbf{A}}_{\mathrm{p}} \mathbf{G}_{\mathbf{J}}-\mathbf{I}_{5}
$$

where $\mathbf{I}_{n}$ is the $n \times n$ identity matrix, $\mathbf{0}$ is a zero matrix of proper size, $\mathbf{G}_{\mathbf{J}}=\mathbf{Z}_{\mathrm{J}}^{-1}=\operatorname{diag}\left[G_{1}, \ldots, G_{5}\right]$ is a diagonal matrix containing inverse reference one-port resistances, $\widetilde{\mathbf{X}}_{0}$ is the $4 \times 4$ matrix obtained by removing the $k$ th column $(1 \leq k \leq 5)$ and the $k$ th row of matrix $\mathbf{X}_{0}$ given by

$$
\mathbf{X}_{0}=\left[\begin{array}{ccccc}
G_{4}+G_{5} & -G_{5} & -G_{4} & 0 & -1 \\
-G_{5} & G_{1}+G_{2}+G_{5} & -G_{2} & -G_{1} & 0 \\
-G_{4} & -G_{2} & G_{2}+G_{3}+G_{4} & -G_{3} & +1 \\
0 & -G_{1} & -G_{3} & G_{1}+G_{3} & 0 \\
+1 & 0 & 0 & -1 & 0
\end{array}\right],
$$

while $\widetilde{\mathbf{A}}_{\mathrm{p}}$ is the $3 \times 5$ matrix obtained by removing the $k$ th row of matrix $\mathbf{A}_{\mathrm{p}}$ given by

$$
\mathbf{A}_{\mathrm{p}}=\left[\begin{array}{rrrrr}
0 & 0 & 0 & -1 & -1 \\
-1 & -1 & 0 & 0 & 1 \\
0 & 1 & 1 & 1 & 0 \\
1 & 0 & -1 & 0 & 0
\end{array}\right]
$$
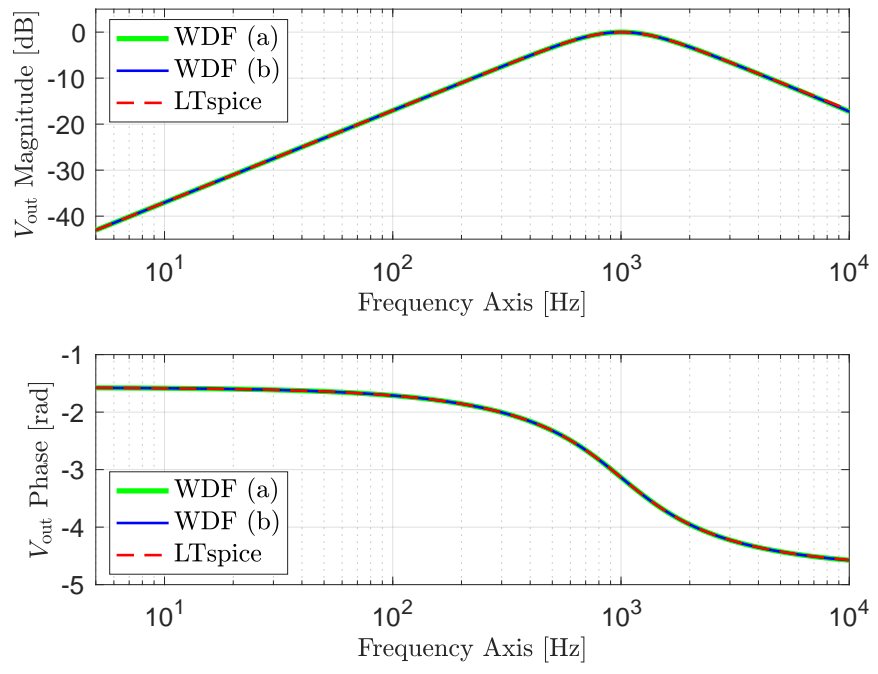

Fig. 13. Active band-pass filter. Comparison between a WDF implementation based on the proposed approach (named "WDF (a)" in the legend), a WDF implementation based on the approach in [17] (named "WDF (b)" in the legend), and an LTspice implementation. The upper plot is the amplitude response of the $V_{\text {out }}$ signal, while the lower plot is the phase response.

The two WDFs in Fig. 11 can be both implemented in a fully explicit fashion without resorting to iterative solvers. We verified that both the WDFs accurately match the behavior of the reference circuit, as confirmed by Fig. 13, which shows a comparison between their frequency response (amplitude and phase) and the frequency response obtained through an LTspice simulation of the reference circuit. As far as the differences of the two WD structures are concerned, the vector implementation in Fig. 11(a) is characterized by a higher degree of modularity than the scalar implementation in Fig. 11(b) As a matter of fact, in the first case the elements (i.e., the one-ports and the opamp) are modeled using WD blocks that are separated from the 7-port WD junction which contains only topological information, while in the second case the opamp model is embedded in the 5port WD junction. Notice that, in case we wanted to change the opamp model (e.g., employing the VCVS-based model in Fig. 12(c) instead of the nullor-based one in Fig. 12(b) in the WD implementation of Fig. 11(a) we would only need to 
change the scattering relation of the two-port WD block (e.g., using eq. (49) in place of eq. (56), while leaving the scattering matrix $\mathbf{S}_{\mathcal{J}_{1}}$ unaltered. Conversely, changing the opamp model in the WD structure of Fig. 11(b) would require to form new matrices $\mathbf{X}_{0}$ and $\mathbf{A}_{\mathrm{p}}$, and recompute the scattering matrix $\mathbf{S}_{\mathcal{R}_{1}}$ from scratch. A further difference is that, despite the size of $\mathbf{S}_{\mathcal{J}_{1}}$ being larger than the size of $\mathbf{S}_{\mathcal{R}_{1}}(7 \times 7$ vs $5 \times 5)$, the formation of $\mathbf{S}_{\mathcal{R}_{1}}$ requires the inversion of the $4 \times 4$ matrix $\widetilde{\mathbf{X}}_{0}$, while the formation of $\mathbf{S}_{\mathcal{J}_{1}}$ requires the inversion of the $3 \times 3$ matrix $\left(\mathbf{Q Z}_{\mathrm{J}}^{-1} \mathbf{Q}^{T}\right)$, as reported in Table $\mathrm{I}$. This makes the WDF in Fig. 11(a) more suitable in scenarios in which the circuit parameters are time-varying and the scattering matrix of the junction needs to be recomputed.

\section{B. WD Implementations of the Phono Preamplifier Circuit with Two LM4562 Opamps}

The phono preamp circuit in Fig. 14 is characterized by the following parameters: $C_{\mathrm{p} 1}=10 \mathrm{pF}, R_{\mathrm{p} 1}=47 \mathrm{k} \Omega, R_{\mathrm{q} 1}=150$ $\Omega, R_{\mathrm{f} 1}=3320 \Omega, R_{\mathrm{con}}=27009 \Omega, C_{\mathrm{pl} 2}=27.2 \mathrm{nF}$, $R_{\mathrm{p} 2}=3930 \Omega, C_{\mathrm{pr} 2}=80 \mathrm{nF}, R_{\mathrm{q} 2}=150 \Omega, R_{\mathrm{f} 2}=3320$ $\Omega$ and $R_{\text {out }}=100 \mathrm{k} \Omega$. The opamps $U_{\mathrm{f} 1}$ and $U_{\mathrm{f} 2}$ are two Texas Instrument LM4562 audio operational amplifiers and are described using the model in Fig. 12(d). The model parameters are chosen according to the LM4562 datasheet; $R_{\text {opOut } 1}=R_{\text {opOut } 2}=1000 \mathrm{M} \Omega, R_{\text {opIn1 }}=R_{\text {opIn2 }}=0.01 \Omega$, $A_{01}=A_{02}=140$. We consider the two WD implementations in Fig. 15

The WD structure in Fig. 15(a) is characterized by series/parallel adaptors $\left(\mathcal{P}_{1}, \mathcal{P}_{2}, \mathcal{S}_{1}\right)$ designed according to traditional WDF principles [1] and a 12-port WD topological junction $\mathcal{J}_{2}$, based on the vectorial definition of waves at the ports of the opamps. The scattering matrix $\mathbf{S}_{\mathcal{J}_{2}}$ is derived by following the approach of Section $\mathrm{X}$ and using eq. 65, where the block-diagonal matrix of free parameters is $\mathbf{Z}_{\mathrm{J}}=\operatorname{blkdiag}\left[\mathbf{Z}_{1,2}, \mathbf{Z}_{3,4}, Z_{5}, Z_{6}, \ldots, Z_{12}\right]$ and

$$
\mathbf{Q}=\left[\begin{array}{cccccccccccc}
1 & 0 & 0 & 0 & 0 & 0 & 1 & 0 & 0 & 0 & 0 & 0 \\
-1 & 1 & 0 & 0 & 0 & -1 & 0 & 1 & 0 & 0 & 0 & 0 \\
0 & -1 & 0 & 0 & 0 & 1 & 0 & 0 & 1 & 0 & 0 & 0 \\
0 & 0 & 1 & 0 & 0 & 1 & 0 & 0 & 0 & 1 & 0 & 0 \\
0 & 0 & -1 & 0 & 1 & 0 & 0 & 0 & 0 & 0 & 1 & 0 \\
0 & 0 & 0 & 1 & -1 & 0 & 0 & 0 & 0 & 0 & 0 & 1
\end{array}\right]
$$

According to 51, WD opamp models are adapted by setting $\mathbf{Z}_{1,2}=\left[\begin{array}{cc}R_{\text {opIn1 }} & 0 \\ A_{01} R_{\text {opIn1 }} & R_{\text {opOut } 1}\end{array}\right], \mathbf{Z}_{3,4}=\left[\begin{array}{cc}R_{\text {opIn2 }} & 0 \\ A_{02} R_{\text {opIn2 }} & R_{\text {opOut2 }}\end{array}\right]$

The WD structure in Fig. 15(b), instead, is designed by following the approach in [17] based on a traditional scalar port-wise definition of waves. The implementation employs a WD 12-port nonreciprocal junction $\mathcal{R}_{2}$. For computability reasons, in fact, the two controlled sources of the two opamp models are embedded in $\mathcal{R}_{2}$. The scattering matrix of $\mathcal{R}_{2}$ is

$$
\mathbf{S}_{\mathcal{R}_{2}}=2 \widetilde{\mathbf{A}}_{\mathrm{p}}^{T}\left[\begin{array}{ll}
\mathbf{I}_{8} & \mathbf{0}
\end{array}\right] \widetilde{\mathbf{X}}_{0}^{-1}\left[\begin{array}{ll}
\mathbf{I}_{8} & \mathbf{0}
\end{array}\right]^{T} \widetilde{\mathbf{A}}_{\mathrm{p}} \mathbf{G}_{\mathbf{J}}-\mathbf{I}_{12}
$$

where $\mathbf{G}_{\mathbf{J}}=\mathbf{Z}_{\mathbf{J}}^{-1}=\operatorname{diag}\left[G_{1}, \ldots, G_{12}\right], \widetilde{\mathbf{X}}_{0}$ is the $10 \times 10$ matrix obtained by removing the $k$ th column $(1<k \leq 11)$ and the $k$ th row of matrix $\mathbf{X}_{0}$ reported in Fig. 16 , while $\widetilde{\mathbf{A}}_{\mathrm{p}}$
TABLE I

SiZE OF THE BIGGEST MATRIX TO BE INVERTED

\begin{tabular}{c|c|c}
\hline Circuit & Approach in $[\mathbf{1 7}]$ & Proposed Approach \\
\hline Active Band-Pass Filter & $4 \times 4$ & $3 \times 3$ \\
\hline Phono Preamplifier & $10 \times 10$ & $6 \times 6$ \\
\hline
\end{tabular}

is the $8 \times 12$ matrix obtained removing the $k$ th row of matrix $\mathbf{A}_{\mathrm{p}}$ given by

$\mathbf{A}_{\mathrm{p}}=\left[\begin{array}{cccccccccccc}-1 & -1 & -1 & -1 & -1 & 0 & 0 & 0 & 0 & 0 & 0 & 0 \\ 1 & 0 & 0 & 0 & 0 & 1 & 0 & 0 & 0 & 0 & 0 & 0 \\ 0 & 1 & 0 & 0 & 0 & -1 & 0 & 1 & 0 & 0 & 0 & 0 \\ 0 & 0 & 0 & 0 & 0 & 0 & -1 & 0 & 0 & 0 & 0 & 0 \\ 0 & 0 & 0 & 0 & 0 & 0 & 1 & -1 & -1 & 0 & 0 & 0 \\ 0 & 0 & 1 & 0 & 0 & 0 & 0 & 0 & 1 & 1 & 0 & 0 \\ 0 & 0 & 0 & 1 & 0 & 0 & 0 & 0 & 0 & -1 & 0 & -1 \\ 0 & 0 & 0 & 0 & 0 & 0 & 0 & 0 & 0 & 0 & -1 & 0 \\ 0 & 0 & 0 & 0 & 1 & 0 & 0 & 0 & 0 & 0 & 1 & 1\end{array}\right]$.

The four resistances $\left(R_{\text {opIn } 1}, R_{\text {opOut } 2}, R_{\text {opIn } 2}, R_{\text {opOut } 2}\right)$ of the opamp models are connected to the junction $\mathcal{R}_{2}$.

The two WDFs in Fig. 15 can be both implemented in a fully explicit fashion without resorting to iterative solvers and they are equally accurate as shown in Fig. 17. Also in this case, the proposed vector implementation in Fig. 15(a) is characterized by a higher degree of modularity as the opamp elements and the topological connections are modeled with separated WD blocks. Moreover, the computational cost required for the formation of $\mathbf{S}_{\mathcal{J}_{2}}$ is lower than the cost required for the formation of $\mathbf{S}_{\mathcal{R}_{2}}$, despite they both describe a 12-port WD junction. In fact, the most expensive operation involved in the derivation of $\mathbf{S}_{\mathcal{R}_{2}}$ is the inversion of the $10 \times 10$ matrix $\widetilde{\mathbf{X}}_{0}$, while, in the derivation of $\mathbf{S}_{\mathcal{J}_{2}}$, it is the inversion of the $6 \times 6$ matrix $\left(\mathbf{Q Z} \mathbf{Z}^{-1} \mathbf{Q}^{T}\right)$, as reported in Table I

\section{CONCLUSIONS AND Future WORK}

In this manuscript we generalized the port-wise definition of wave variables, employed in traditional WDFs, to a vector definition, which is particularly useful for modeling circuits with two-port elements, e.g., ideal and resistive controlled sources, gyrators and nullors. The proposed vector wave definition allows us to overcome several computability issues, such as the delay-free-loops that are formed when connecting a two-port to a topological junction, which are unavoidable in WDFs based on scalar waves. This is done while preserving the modularity of traditional WDFs, in which the topological connections and the elements are modeled separately, i.e., using different WD scattering blocks. Moreover, as discussed in Section XI, the proposed approach generally requires the inversion of smaller matrices for the computation of the scattering matrices of multi-port junctions.

The presented WDF modeling approach can be extended to circuits containing $M$-port elements, with $M \geq 2$, adopting the following vector definition of waves, that generalizes (3),

$$
\mathbf{a}_{1: M}=\mathbf{v}_{1: M}+\mathbf{Z}_{1: M} \mathbf{i}_{1: M}, \quad \mathbf{b}_{1: M}=\mathbf{v}_{1: M}-\mathbf{Z}_{1: M} \mathbf{i}_{1: M},
$$

where $\mathbf{a}_{1: M}$ and $\mathbf{b}_{1: M}$ are $M \times 1$ vectors of incident waves and reflected waves, respectively, $\mathbf{v}_{1: M}$ and $\mathbf{i}_{1: M}$ are vectors collecting port voltages and port currents of the $M$-port, respectively, while $\mathbf{Z}_{1: M}$ is a $M \times M$ full-rank matrix of 


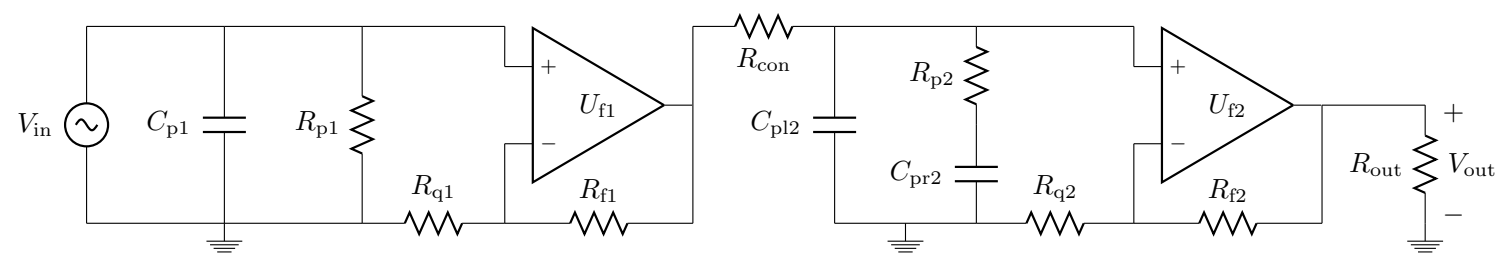

Fig. 14. Phono preamplifier circuit.

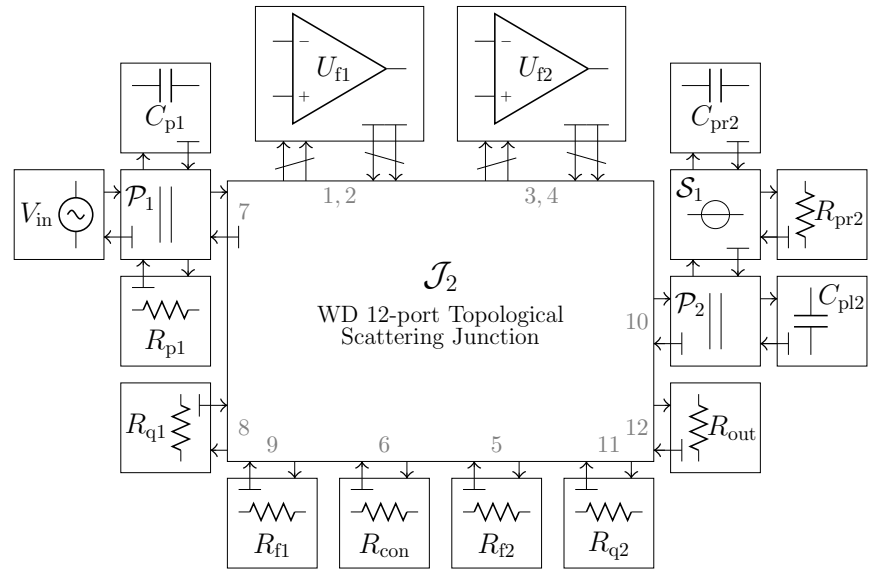

(a)

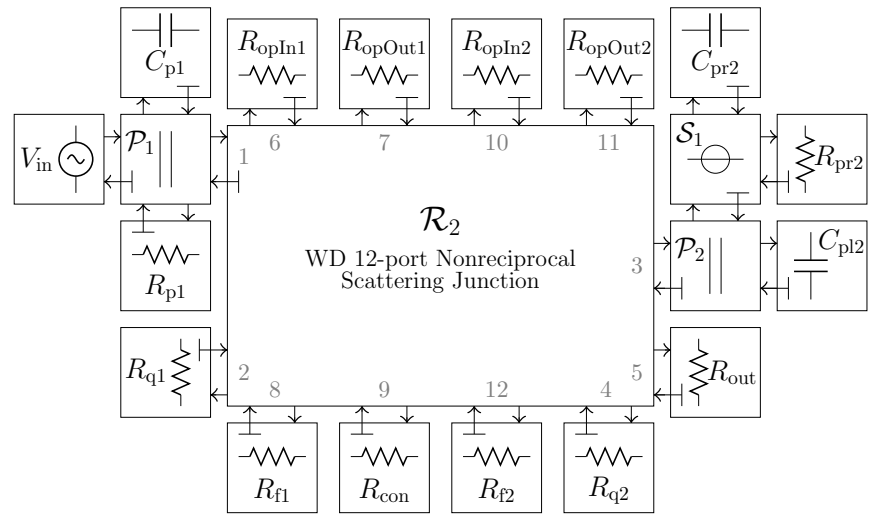

(b)

Fig. 15. WDF realizations of the circuit in Fig. 14

free parameters. An in-depth analysis of the properties of WD models of relevant $M$-ports based on such a vector definition of waves will be provided in future works.

The proposed approach could be combined with the macromodeling method for linear multi-ports presented in [32].

As a further development, it is worth applying vector WDFs to the implementation of circuits with nonlinear multi-port elements [33]-[35], such as transistors or nonlinear amplifiers.

\section{REFERENCES}

[1] A. Fettweis, "Wave digital filters: Theory and practice," Proc. IEEE, vol. 74, no. 2, pp. 270-327, Feb. 1986.

[2] A. Fettweis, "Pseudopassivity, sensitivity, and stability of wave digital filters," IEEE Trans. Circuit Theory, vol. 19, pp. 668-673, Nov. 1972.

[3] A. Bernardini, P. Maffezzoni, and A. Sarti, "Linear multistep discretization methods with variable step-size in nonlinear wave digital structures for virtual analog modeling," IEEE/ACM Trans. Audio, Speech, Language Process, vol. 27, no. 11, pp. 1763-1776, Nov 2019.
[4] A. Fettweis and K. Meerkötter, "On adaptors for wave digital filters," IEEE Trans. Acoust., Speech, Signal Process., vol. 23, pp. 516-525, Dec. 1975.

[5] K. Meerkötter and R. Scholz, "Digital simulation of nonlinear circuits by wave digital filter principles," in IEEE Int. Symp. Circuits Syst., vol. 1, June 1989 , pp. $720-723$.

[6] A. Sarti and G. De Sanctis, "Systematic methods for the implementation of nonlinear wave-digital structures," IEEE Trans. Circuits Syst. I, Reg. Papers, vol. 56, pp. 460-472, Feb. 2009.

[7] G. De Sanctis and A. Sarti, "Virtual analog modeling in the wavedigital domain," IEEE Transactions on Audio, Speech and Language Processing, vol. 18, no. 4, pp. 715-727, May 2010.

[8] A. Bernardini and A. Sarti, "Dynamic adaptation of instantaneous nonlinear bipoles in wave digital networks," in Proc. 24th Eur. Signal Process. Conf. (EUSIPCO), Budapest, Hungary, Aug. 2016, pp. 10381042.

[9] M. J. Olsen, K. J. Werner, and J. O. Smith III, "Resolving grouped nonlinearities in wave digital filters using iterative techniques," in Proc. 19th Int. Conf. Digital Audio Effects, Brno, Czech Republic, Sept. 2016, pp. 279-286.

[10] A. Bernardini, P. Maffezzoni, L. Daniel, and A. Sarti, "Wave-based analysis of large nonlinear photovoltaic arrays," IEEE Trans. Circuits Syst. I: Reg. Papers, vol. 65, no. 4, pp. 1363-1376, Apr. 2018.

[11] A. Bernardini, A. Sarti, P. Maffezzoni, and L. Daniel, "Wave digitalbased variability analysis of electrical mismatch in photovoltaic arrays," in Proc. IEEE Int. Symp. Circuits Syst. (ISCAS), Florence, Italy, May 2018.

[12] A. Bernardini, K. J. Werner, P. Maffezzoni, and A. Sarti, "Wave digital modeling of the diode-based ring modulator," in Proc. 144th Audio Engineering Society (AES) Conv., Milan, Italy, May 2018, id. \#10015.

[13] T. Schwerdtfeger and A. Kummert, "Nonlinear circuit simulation by means of Alfred Fettweis' wave digital principles," IEEE Circuits and Systems Magazine, vol. 19, no. 1, pp. 55-C3, Firstquarter 2019.

[14] A. Proverbio, A. Bernardini, and A. Sarti, "Toward the wave digital realtime emulation of audio circuits with multiple nonlinearities," in Proc. 28th Eur. Signal Process. Conf. (EUSIPCO), Amsterdam, NL, Jan. 2021, pp. 151-155.

[15] G. O. Martens and K. Meerkötter, "On N-port adaptors for wave digital filters with application to a bridged-tee filter," in Proc. IEEE Int. Symp. Circuits Syst. (ISCAS), Munich, Germany, Apr. 1976, pp. 514-517.

[16] G. O. Martens and H. H. Lê, "Wave digital adapters for reciprocal second-order sections," IEEE Trans. Circuits Syst., vol. 25, no. 12, pp. 1077-1083, Dec. 1978.

[17] K. J. Werner, A. Bernardini, J. O. Smith, and A. Sarti, "Modeling circuits with arbitrary topologies and active linear multiports using wave digital filters," IEEE Trans. Circuits Syst. I: Reg. Papers, vol. 65, no. 12, pp. 4233-4246, Dec 2018.

[18] A. Bernardini, K. J. Werner, J. O. Smith III, and A. Sarti, "Generalized wave digital filter realizations of arbitrary reciprocal connection networks," IEEE Trans. Circuits Syst. I: Reg. Papers, vol. 66, no. 2, pp. 694-707, Feb 2019.

[19] D. Fränken, J. Ochs, and K. Ochs, "Generation of wave digital structures for networks containing multiport elements," IEEE Trans. Circuits Syst. I: Reg. Papers, vol. 52, no. 3, pp. 586-596, Mar. 2005.

[20] K. J. Werner, J. O. Smith, and J. S. Abel, "Wave digital filters adaptors for arbitrary topologies and multiport linear elements," in Proc. 18th Int. Conf. Digital Audio Effects, Trondheim, Norway, Nov.-Dec. 2015.

[21] K. J. Werner, W. R. Dunkel, M. Rest, M. J. Olsen, and J. O. Smith, "Wave digital filter modeling of circuits with operational amplifiers," in Proc. 24th Eur. Signal Process. Conf. (EUSIPCO), Budapest, Hungary, Aug. 2016, pp. 1033-1037.

[22] M. Verasani, A. Bernardini, and A. Sarti, "Modeling Sallen-Key audio filters in the wave digital domain," in Proc. IEEE Int. Conf. Acoust., Speech Signal Process., New Orleans, LA, Mar. 2017, pp. 431-435. 

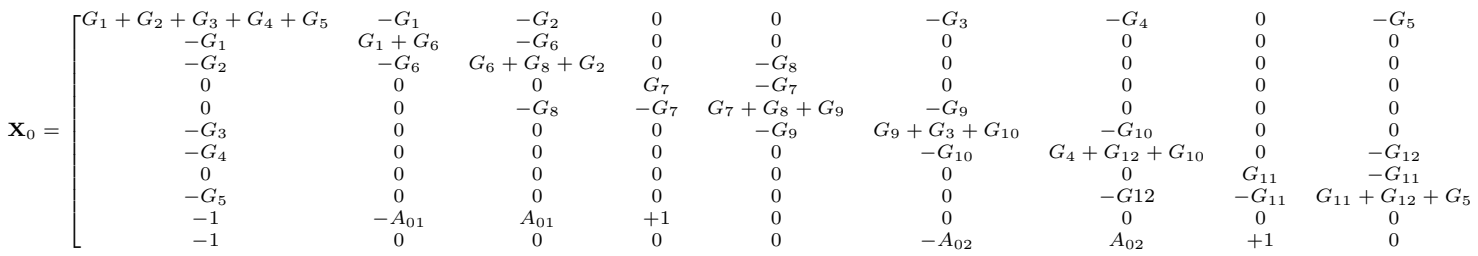

$\left.\begin{array}{cc}-1 & -1 \\ 0 & 0 \\ 0 & 0 \\ +1 & 0 \\ 0 & 0 \\ 0 & 0 \\ 0 & 0 \\ 0 & +1 \\ 0 & 0 \\ 0 & 0 \\ 0 & 0\end{array}\right]$

Fig. 16. Phono preamplifier. Nodal matrix $\mathbf{X}_{0}$ needed for the formation of $\mathbf{S}_{\mathcal{R}_{2}}$.
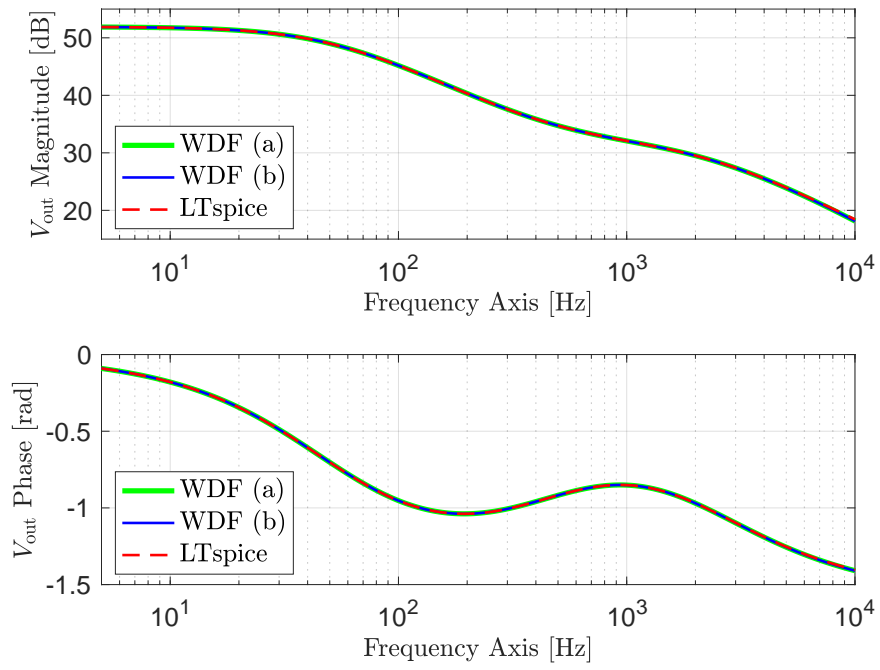

Fig. 17. Phono preamplifier. Comparison between a WDF implementation based on the proposed approach (named "WDF (a)" in the legend), a WDF implementation based on the approach in [17] (named "WDF (b)" in the legend), and an LTspice implementation. The upper plot is the amplitude response of the $V_{\text {out }}$ signal, while the lower plot is the phase response.

[23] Ó. Bogason and K. J. Werner, "Modeling circuits with operational transconductance amplifiers using wave digital filters," in Proc. 20th Int. Conf. Digital Audio Effects, Edinburgh, UK, Sept. 2017, pp. 130-137.

[24] H. Carlin, "Singular network elements," IEEE Transactions on Circuit Theory, vol. 11, no. 1, pp. 67-72, March 1964.

[25] L. Chua, C. Desoer, and E. Kuh, Linear and Nonlinear Circuits. New York, NY: McGraw-Hill, 1987.

[26] G. Kubin, "Wave digital filters: Voltage, current, or power waves?" in Proc. IEEE Int. Conf. Acoust., Speech Signal Process., Tampa, Florida, Apr. 1985, pp. 69-72.

[27] S. Bilbao, Wave and Scattering Methods for Numerical Simulation, 1st ed. New York: John Wiley \& Sons, Oct. 2004.

[28] K. J. Werner, "Virtual analog modeling of audio circuitry using wave digital filters," Ph.D. diss., Department of Music, Stanford Univ., CA, 2016.

[29] A. Bernardini and A. Sarti, "Biparametric wave digital filters," IEEE Trans. Circuits Syst. I: Reg. Papers, vol. 64, no. 7, pp. 1826-1838, July 2017.

[30] A. Fettweis, "Reciprocity, inter-reciprocity, and transposition in wave digital filters," Int. J. Circ. Theor. Appl., vol. 1, pp. 323-337, 1973.

[31] K. Meerkötter and D. Fränken, "Digital realization of connection networks by voltage-wave 2-port adapters," Arch. Elek. Übertragung. (AË̈, Int. J. Electron. Commun.), vol. 50, no. 6, pp. 362-367, 1996.

[32] P. Belforte, D. Spina, L. Lombardi, G. Antonini, and T. Dhaene, "Automated framework for time-domain piecewise-linear fitting method based on digital wave processing of $s$-parameters," IEEE Trans. Circuits Syst. I: Reg. Papers, vol. 67, no. 1, pp. 235-248, 2020.

[33] A. Bernardini, K. J. Werner, A. Sarti, and J. O. Smith III, "Modeling nonlinear wave digital elements using the Lambert function," IEEE Trans. Circuits Syst. I: Reg. Papers, vol. 63, no. 8, pp. 1231-1242, Aug. 2016.

[34] A. Bernardini, K. J. Werner, A. Sarti, and J. O. Smith III, "Modeling a class of multi-port nonlinearities in wave digital structures," in Proc. 23rd Eur. Signal Process. Conf. (EUSIPCO), Nice, France, Aug. 2015, pp. 669-673.

[35] A. Bernardini, A. E. Vergani, and A. Sarti, "Wave digital modeling of nonlinear 3-terminal devices for virtual analog applications," Circuits, Systems, and Signal Processing, vol. 39, pp. 3289-3319, Jan. 2020.

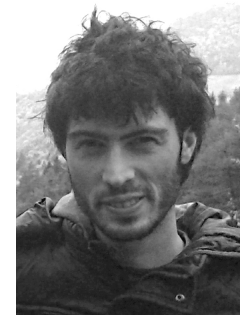

Alberto Bernardini (S'16-M'19) received his B.S. degree from University of Bologna, Italy, in 2012 and his M.S. degree (cum laude) from Politecnico di Milano, Italy, in 2015, both in Computer Engineering. In 2019, he received his Ph.D. degree (cum laude) in Information Engineering from Politecnico di Milano, Italy, where he is currently a postdoctoral researcher. His main research interests are audio signal processing and modeling of nonlinear systems. He authored over 20 publications in international journals and proceedings of international conferences. He is also the first author of an international patent.

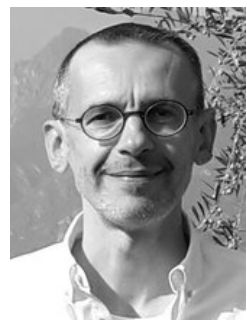

Paolo Maffezzoni (M'08-SM'15) received the Laurea degree (summa cum laude) in electrical engineering from Politecnico di Milano, Italy, in 1991 and the $\mathrm{Ph} . \mathrm{D}$. degree in electronic instrumentation from Universita' di Brescia, Italy, in 1996 . He is currently a Full Professor of electrical engineering with Politecnico di Milano, Italy. His research interests include the development of advanced computational methods for the simulation/design of nonlinear circuits and systems, oscillators, phase-domain modeling, probabilistic analysis and unconventional computing with applications to electronics and power systems. He has published about 140 research papers in international journals and conferences. He served as an Associate Editor for the IEEE Transactions on Computer-Aided Design of Integrated Circuits and Systems and the IEEE Transactions on Circuits and Systems I: Regular Papers. 


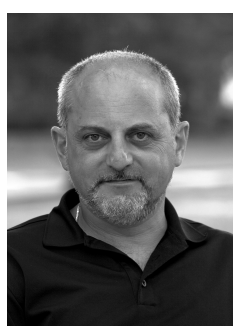

Augusto Sarti (M'04-SM'13) received his Ph.D. in Information Engineering from the University of Padova, Italy, in 1993, with a joint graduate program with the University of California, Berkeley. In 1993 , he joined the Politecnico di Milano (PoliMI), Italy, where he is currently a Full Professor. From 2013 to 2017 he held a professorship at the University of California, Davis. At PoliMI he currently coordinates the research activities of the Musical Acoustics Lab and the Sound and Music Computing Lab, and the M.Sci. program in Music and Acoustic Engineering. He has coauthored over 300 scientific publications on international journals and congresses and numerous patents in the multimedia signal processing area. His current research interests are in the area of audio and acoustic signal processing, with particular focus on audio and acoustic signal processing; music information retrieval; and musical acoustics. He served two terms with the IEEE Technical Committee on Audio and Acoustics Signal Processing. He also served as Associate Editor of IEEE/ACM Tr. on Audio Speech and Language Processing, and as Senior Area Editor of IEEE Signal Processing Letters. He is currently serving in the EURASIP board of directors. 